\title{
BISIMILARITY ON BASIC PROCESS ALGEBRA IS IN 2-EXPTIME (AN EXPLICIT PROOF)
}

\author{
PETR JANČAR
}

Technical University Ostrava (FEI VŠB-TUO), Czech Rep.

e-mail address: petr.jancar@vsb.cz

\begin{abstract}
Burkart, Caucal, Steffen (1995) showed a procedure deciding bisimulation equivalence of processes in Basic Process Algebra (BPA), i.e. of sequential processes generated by context-free grammars. They improved the previous decidability result of Christensen, Hüttel, Stirling (1992), since their procedure has obviously an elementary time complexity and the authors claim that a close analysis would reveal a double exponential upper bound. Here a self-contained direct proof of the membership in 2-ExpTime is given. This is done via a Prover-Refuter game which shows that there is an alternating Turing machine deciding the problem in exponential space. The proof uses similar ingredients (size-measures, decompositions, bases) as the previous proofs, but one new simplifying factor is an explicit addition of infinite regular strings to the state space. An auxiliary claim also shows an explicit exponential upper bound on the equivalence level of nonbisimilar normed BPA processes.

The importance of clarifying the 2-ExpTime upper bound for BPA bisimilarity has recently increased due to the shift of the known lower bound from PSpace (Srba, 2002) to ExpTime (Kiefer, 2012).
\end{abstract}

\section{INTRODUCTION}

The classical language equivalence problems in automata theory have their counterparts in the bisimulation equivalence problems in process theory. The computational complexity of bisimulation equivalence is still not fully settled even for fundamental classes, one of them being the class of Basic Process Algebra (BPA) processes, i.e. of sequential processes generated by context-free grammars. This concrete research topic started with a result by Baeten, Bergstra, Klop [1] who showed decidability in the normed BPA case (where each nonterminal of the underlying context-free grammar derives some terminal word). Christensen, Hüttel, Stirling [8] extended the decidability result to the whole BPA class, and Burkart, Caucal, Steffen [6] (see also [5]) showed a procedure with an elementary

2012 ACM CCS: [Theory of computation]: Formal languages and automata theory-FormalismsRewrite systems; Semantics and reasoning-Program semantics-Action semantics; Logic-Logic and verification.

Key words and phrases: bisimulation equivalence, basic process algebra, complexity.

The work was supported by the Czech Grant Agency (GAČR:P202/11/0340) and partly by the European Regional Development Fund in the IT4Innovations Centre of Excellence project (CZ.1.05/1.1.00/02.0070).

DOI:10.2168/LMCS-9(1:10)2013

(C) P. Jančar

(C) Creative Commons 
complexity, claiming that a close analysis would demonstrate a double exponential upper bound. We also note that the normed case was subsequently shown to be in PTIME [10] (see [9] for the most recent improvement of complexity).

Regarding the lower bounds for the (full) BPA problem, Srba [19] showed PSPACEhardness, and Kiefer [15] recently shifted this to ExPTime-hardness (using the ExPTIMEcompleteness of countdown games [14]); he thus also strengthened the lower bound results known for (visibly) pushdown processes [16], 20] and for weak bisimilarity [17]. This was a bit surprising since the bisimulation equivalence problem for related classes of basic parallel processes (generated by commutative context-free grammars) and of one-counter processes were shown PSPACE-complete [11, [3. The mentioned shift of the lower bound is a natural impulse for looking at the complexity again; confirming the upper bound which has been a bit vaguely stated in the literature becomes more important.

Here we show a direct self-contained proof of the fact that BPA bisimilarity is indeed in 2-ExpTIME. This is done via a Prover-Refuter game which shows that there is an alternating Turing machine deciding the problem in exponential space. The proof uses similar ingredients (size-measures, decompositions, bases) as the previous proofs, though in somewhat different forms; a new factor is an explicit addition of infinite regular strings to the state space. On the whole, the proof confirms the previously claimed upper bound, simplifies several technical aspects, and it might also shed some new light on the structural decomposition approach for deciding bisimilarity. An auxiliary claim also shows an exponential upper bound on the equivalence level of nonbisimilar normed BPA processes; such a bound seems to have been only implicit in the previous works.

Section 2 recalls the notion of regular strings, defines the bisimilarity problem for BPA and states the main result. Section 3 then shows a proof. It recalls some simple notions and observations, including the congruence properties and decompositions, and then a ProverRefuter game is defined; it will be obvious that Refuter has a winning strategy for negative instances. The above mentioned exponential upper bound on the equivalence level of nonbisimilar normed BPA processes, which is used to show that Prover has a winning strategy for positive instances, is highlighted in Section 4. Section 5 adds some further remarks.

\section{Preliminaries}

Let $\mathbb{N}=\{0,1,2, \ldots\}$. For a finite set $\mathcal{C}, \operatorname{card}(\mathcal{C})$ is the number of elements of $\mathcal{C}$, and $\mathcal{C}^{*}$ is the set of finite sequences of elements of $\mathcal{C}$, also called strings or words over $\mathcal{C}$. By $\varepsilon$ we denote the empty sequence and by $|w|$ the length of $w \in \mathcal{C}^{*}$. By $\mathcal{C}^{\omega}$ we denote the set of infinite strings over $\mathcal{C}$, i.e. the set of mappings $\mathbb{N} \rightarrow \mathcal{C}$. By $u v$ we denote the concatenation of strings $u, v$. For technical convenience, we might write $u v$ even when $u$ is infinite but then $u v$ is implicitly identified with $u$. We put $u^{0}=\varepsilon$ and $u^{i+1}=u u^{i}$ (where $i \in \mathbb{N}$ ). By $u^{\omega}$ we denote the string $u u u \cdots ; u^{\omega}=u$ when $u$ is infinite, and $\varepsilon^{\omega}=\varepsilon$. If $w=u v$ then $u$ is a prefix of $w$; if $u$ is finite then $v$ is a suffix of $w$.

Regular strings. A regular string over $\mathcal{C}$ is either a finite string (an element of $\mathcal{C}^{*}$ ) or an infinite string (an element of $\mathcal{C}^{\omega}$ ) of the form $\beta \gamma \gamma \gamma \cdots=\beta \gamma^{\omega}$ where $\beta, \gamma \in \mathcal{C}^{*}$ and $\gamma \neq$ $\varepsilon$. (Such infinite strings are also called ultimately periodic words.) We do not consider nonregular strings.

One infinite regular string can have more "lasso" presentations, as shown by the example

$$
B A A(B B A B B A B B A)^{\omega}=B A(A B B)^{\omega} .
$$


The second presentation is the canonical one, since it has the shortest cycle $(A B B)$ and the shortest prefix $(B A)$. We now make this standard notion precise, while also recalling some standard facts which will be used later.

For $\alpha \in \mathcal{C}^{*}$ we put $\operatorname{swAP}(\alpha)=\{\gamma \beta \mid \beta \gamma=\alpha\}$.

Proposition 2.1. If $\beta_{1}\left(\gamma_{1}\right)^{\omega}=\beta_{2}\left(\gamma_{2}\right)^{\omega}$ then $\left(\gamma_{2}\right)^{\omega}=\left(\gamma_{1}^{\prime}\right)^{\omega}$ for some $\gamma_{1}^{\prime} \in \operatorname{SWAP}\left(\gamma_{1}\right)$.

Proof. Since $\beta_{1} \gamma_{1} \gamma_{1} \gamma_{1} \cdots=\beta_{2} \gamma_{2} \gamma_{2} \gamma_{2} \ldots$, we obviously must have $\gamma_{2} \gamma_{2} \gamma_{2} \ldots=\delta \gamma_{1} \gamma_{1} \gamma_{1} \ldots$ for a suffix $\delta$ of $\gamma_{1}$; let $\gamma_{1}=\delta^{\prime} \delta$. Hence $\left(\gamma_{2}\right)^{\omega}=\delta\left(\delta^{\prime} \delta\right)^{\omega}=\left(\delta \delta^{\prime}\right)^{\omega}$.

Lemma 2.2. Each regular string $\alpha$ has the unique prefix $\alpha_{p}$ and the unique cycle $\alpha_{c}$ such that $\alpha=\alpha_{p}\left(\alpha_{c}\right)^{\omega}$ and, moreover, $\alpha=\beta \gamma^{\omega}$ implies $|\beta| \geq\left|\alpha_{p}\right|$ and $|\gamma| \geq\left|\alpha_{c}\right|$ (if $\beta$ is finite).

Proof. Suppose $\alpha=\beta_{1}\left(\gamma_{1}\right)^{\omega}=\beta_{2}\left(\gamma_{2}\right)^{\omega}$. Using Prop. 2.1, we get

$$
\alpha=\beta_{1}\left(\gamma_{1}\right)^{\omega}=\beta_{1}\left(\gamma_{2}^{\prime}\right)^{\omega}=\beta_{2}\left(\gamma_{2}\right)^{\omega}=\beta_{2}\left(\gamma_{1}^{\prime}\right)^{\omega}
$$

for some $\gamma_{2}^{\prime} \in \operatorname{SWAP}\left(\gamma_{2}\right)$ and $\gamma_{1}^{\prime} \in \operatorname{SWAP}\left(\gamma_{1}\right)$. It is thus obvious that $\alpha=\beta \gamma^{\omega}$ where $|\beta|=\min \left\{\left|\beta_{1}\right|,\left|\beta_{2}\right|\right\}$ and $|\gamma|=\min \left\{\left|\gamma_{1}\right|,\left|\gamma_{2}\right|\right\}$. The claim thus follows easily.

We call $\alpha_{p}\left(\alpha_{c}\right)^{\omega}$ the canonical presentation of $\alpha$ (where $\alpha_{p}=\alpha$ and $\alpha_{c}=\varepsilon$ when $\alpha$ is finite). It is useful to note that the (canonical) cycle of a regular string is insensitive to any change of a finite prefix, up to swapping:

Proposition 2.3. For any finite $\beta_{1}, \beta_{2}$ and any (regular) $\alpha$ we have $\left(\beta_{2} \alpha\right)_{c} \in \operatorname{SWAP}\left(\left(\beta_{1} \alpha\right)_{c}\right)$.

Proof. We have $\beta_{1} \alpha=\left(\beta_{1} \alpha\right)_{p}\left(\left(\beta_{1} \alpha\right)_{c}\right)^{\omega}=\beta_{1} \alpha_{p}\left(\alpha_{c}\right)^{\omega}$; hence $\left|\left(\beta_{1} \alpha\right)_{c}\right| \leq\left|\alpha_{c}\right|$ (by Lemma 2.2). On the other hand, $\alpha=\gamma_{1}\left(\left(\beta_{1} \alpha\right)_{c}\right)^{\omega}$ for some finite $\gamma_{1}$, and thus $\left|\alpha_{c}\right| \leq\left|\left(\beta_{1} \alpha\right)_{c}\right|$; hence $\left|\left(\beta_{1} \alpha\right)_{c}\right|=\left|\alpha_{c}\right|$. Similarly $\alpha=\gamma_{2}\left(\left(\beta_{2} \alpha\right)_{c}\right)^{\omega}$ for some finite $\gamma_{2}$, and we deduce $\left|\left(\beta_{1} \alpha\right)_{c}\right|=$ $\left|\left(\beta_{2} \alpha\right)_{c}\right|$. Since $\gamma_{1}\left(\left(\beta_{1} \alpha\right)_{c}\right)^{\omega}=\gamma_{2}\left(\left(\beta_{2} \alpha\right)_{c}\right)^{\omega}$, by Prop. 2.1 we easily derive that $\left(\beta_{2} \alpha\right)_{c} \in$ $\operatorname{SWAP}\left(\left(\beta_{1} \alpha\right)_{c}\right)$.

We will also (implicitly) use the following simple computational fact.

Proposition 2.4. There is a polynomial-time algorithm which, given finite strings $\beta$ and $\gamma$, finds the canonical prefix $\left(\beta \gamma^{\omega}\right)_{p}$ and the canonical cycle $\left(\beta \gamma^{\omega}\right)_{c}$.

Proof. Even a brute-force approach is sufficient here. We can systematically explore all 3part partitions $\beta \gamma=\delta_{1} \delta_{2} \delta_{3}$. For each of them we can check whether $\delta_{1}\left(\delta_{2}\right)^{\omega}=\beta \gamma^{\omega}$ : for this we must have $\delta_{3}=\left(\delta_{2}\right)^{j} \delta, \delta_{2}=\delta \delta^{\prime}$ and $\left(\delta^{\prime} \delta\right)^{\omega}=\gamma^{\omega}$; the latter holds iff $\left(\delta^{\prime} \delta\right)^{|\gamma|}=\gamma^{\left|\delta^{\prime} \delta\right|}$.

$B P A$ processes. A BPA system is defined as a context-free grammar in Greibach normal form with no starting nonterminal; it is a tuple $\mathcal{G}=(\mathcal{N}, \mathcal{A}, \mathcal{R})$ where $\mathcal{N}, \mathcal{A}, \mathcal{R}$ are finite nonempty sets of nonterminals (or variables), actions (or terminals), and rewriting rules, respectively. The rules in $\mathcal{R}$ are of the form $A \stackrel{a}{\longrightarrow} \alpha$ where $A \in \mathcal{N}, a \in \mathcal{A}, \alpha \in \mathcal{N}^{*}$. For later convenience we assume that for each $A \in \mathcal{N}$ there is at least one rule of the form $A \stackrel{a}{\longrightarrow} \alpha$, i.e., there are no dead nonterminals. (But there may still be nonterminals which do not derive any terminal word in the classical language sense.)

With each BPA system $\mathcal{G}=(\mathcal{N}, \mathcal{A}, \mathcal{R})$ we associate the labelled transition system $($ LTS $)$ $\mathcal{L}_{\mathcal{G}}=\left(\mathcal{S}_{\mathcal{G}}, \mathcal{A},(\stackrel{a}{\longrightarrow})_{a \in \mathcal{A}}\right)$ where $\mathcal{S}_{\mathcal{G}}$ is the set of all regular strings over $\mathcal{N}$, which are also called states or processes. The transition relations $\stackrel{a}{\longrightarrow} \subseteq \mathcal{S}_{\mathcal{G}} \times \mathcal{S}_{\mathcal{G}}$ are defined inductively as follows: if $A \stackrel{a}{\longrightarrow} \alpha$ is a rule in $\mathcal{R}$ then $A \beta \stackrel{a}{\longrightarrow} \alpha \beta$ for any regular string $\beta$. We also define $\stackrel{w}{\longrightarrow}$, for $w \in \mathcal{A}^{*}$, as usual: $\alpha \stackrel{\varepsilon}{\longrightarrow} \alpha$; if $\alpha \stackrel{a}{\longrightarrow} \beta$ and $\beta \stackrel{u}{\longrightarrow} \gamma$ then $\alpha \stackrel{a u}{\longrightarrow} \gamma$. 
Remark. We note that $\mathcal{L}_{\mathcal{G}}$ is generally nondeterministic, since $\mathcal{R}$ can contain rules $A \stackrel{a}{\longrightarrow} \alpha$ and $A \stackrel{a}{\longrightarrow} \beta$ where $\alpha \neq \beta$. We also note that if $\alpha$ is a finite string and $\alpha \stackrel{w}{\longrightarrow} \beta$ then $\beta$ is also finite. The convenience of including also infinite regular strings into $\mathcal{S}_{\mathcal{G}}$ will become clear later.

Bisimilarity problem for BPA. Given $\mathcal{G}=(\mathcal{N}, \mathcal{A}, \mathcal{R})$, with the associated LTS $\mathcal{L}_{\mathcal{G}}=$ $\left(\mathcal{S}_{\mathcal{G}}, \mathcal{A},(\stackrel{a}{\longrightarrow})_{a \in \mathcal{A}}\right)$, we say that $\mathcal{B} \subseteq \mathcal{S}_{\mathcal{G}} \times \mathcal{S}_{\mathcal{G}}$ covers $(\alpha, \beta) \in \mathcal{S}_{\mathcal{G}} \times \mathcal{S}_{\mathcal{G}}$ if for any transition $\alpha \stackrel{a}{\longrightarrow} \alpha^{\prime}$ there is $\beta \stackrel{a}{\longrightarrow} \beta^{\prime}$ such that $\left(\alpha^{\prime}, \beta^{\prime}\right) \in \mathcal{B}$, and for any $\beta \stackrel{a}{\longrightarrow} \beta^{\prime}$ there is $\alpha \stackrel{a}{\longrightarrow} \alpha^{\prime}$ such that $\left(\alpha^{\prime}, \beta^{\prime}\right) \in \mathcal{B}$. For subsets $\mathcal{B}, \mathcal{B}^{\prime}$ of $\mathcal{S}_{\mathcal{G}} \times \mathcal{S}_{\mathcal{G}}$ we say that $\mathcal{B}$ covers $\mathcal{B}^{\prime}$ if $\mathcal{B}$ covers each $(\alpha, \beta) \in \mathcal{B}^{\prime}$. A set $\mathcal{B}$ is a bisimulation if $\mathcal{B}$ covers $\mathcal{B}$. States $\alpha, \beta$ are bisimilar, denoted $\alpha \sim \beta$, if there is a bisimulation $\mathcal{B}$ containing $(\alpha, \beta)$.

The problem BPA-Bisim asks, given $\mathcal{G}$ and two nonterminals $X, Y$, if $X \sim Y$. We will prove the next theorem, assuming a standard encoding of $\mathcal{G}, X, Y$.

Theorem 2.5. BPA-BISIM is in 2-ExPTIME; i.e., there is an algorithm which decides BPA-BISIM and its time complexity is in $O\left(2^{2^{\text {pol(n) }}}\right)$ for a polynomial pol.

\section{Proof of Theorem 2.5}

In Subsection 3.1 we define some useful technical notions and observe their properties. These are variants of the ingredients used in the previous works like [8, 10, 6]. The extensions to regular strings are straightforward but we sketch all the proofs, to be self-contained. Subsection 3.2 then describes the crux of the algorithm, formulated as a Prover-Refuter game. Soundness (meaning that Prover cannot force a win when $X \nsim Y$ ) will be obvious, while completeness (Prover can force a win when $X \sim Y$ ) is shown in Subsection 3.3. the proof of a crucial technical lemma, related to normed BPA processes, is separated in Subsection 3.4,

3.1. Useful notions and their properties. We consider a BPA system $\mathcal{G}=(\mathcal{N}, \mathcal{A}, \mathcal{R})$, with the associated labelled transition system $\mathcal{L}_{\mathcal{G}}=\left(\mathcal{S}_{\mathcal{G}}, \mathcal{A},(\stackrel{a}{\longrightarrow})_{a \in \mathcal{A}}\right)$. We put $\sim_{0}=\mathcal{S}_{\mathcal{G}} \times \mathcal{S}_{\mathcal{G}}$, and let $\sim_{i+1} \subseteq \mathcal{S}_{\mathcal{G}} \times \mathcal{S}_{\mathcal{G}}(i \in \mathbb{N})$ be the set of all pairs covered by $\sim_{i}$. We note that $\alpha \chi_{1} \beta$ iff $\alpha, \beta$ enable different sets of actions.

In the next proposition we also use the convention that $\alpha \beta$ and $\alpha^{\omega}$ are identified with $\alpha$ when $\alpha$ is infinite.

\section{Proposition 3.1.}

(1) The relations $\sim$ and $\sim_{i}$ (for all $i \in \mathbb{N}$ ) are equivalences.

(2) If $\alpha \sim_{i+1} \beta$ then $\alpha \sim_{i} \beta$ (hence $\sim_{0} \supseteq \sim_{1} \supseteq \sim_{2} \supseteq \ldots$ ).

(3) We have $\alpha \sim \beta$ iff $\forall i \in \mathbb{N}: \alpha \sim_{i} \beta$.

(4) If $\alpha \sim_{i} \beta$ and $\gamma \sim_{i} \delta$ then $\alpha \gamma \sim_{i} \beta \delta$. Hence $\sim$ and $\sim_{i}$ are congruences w.r.t. concatenation.

(5) If $\alpha \sim_{i} \gamma \alpha$ and $\gamma \neq \varepsilon$ then $\alpha \sim_{i} \gamma^{\omega}$. (Hence $\alpha \sim \gamma \alpha$ implies $\alpha \sim \gamma^{\omega}$.)

Proof. (1) Bisimilarity, i.e. the relation $\sim$, can be easily shown to be the greatest bisimulation, namely the union of all bisimulations; the equivalence conditions can be easily checked. For relations $\sim_{i}$, the equivalence conditions can be easily established by induction on $i$.

(2) can be also easily established by induction on $i$. 
(3) The inclusion $\bigcap_{i \in \mathbb{N}} \sim_{i} \supseteq \sim$ is trivial. Since $\mathcal{L}_{\mathcal{G}}$ is image finite, i.e., for each pair $\alpha \in \mathcal{S}_{\mathcal{G}}$, $a \in \mathcal{A}$ there are only finitely many $\beta$ such that $\alpha \stackrel{a}{\longrightarrow} \beta$, the set $\bigcap_{i \in \mathbb{N}} \sim_{i}$ can be easily checked to be a bisimulation; therefore $\bigcap_{i \in \mathbb{N}} \sim_{i} \subseteq \sim$.

(4) Our assumption that there is no dead nonterminal $A \in \mathcal{N}$ implies $\varepsilon \sim_{1} \alpha$ iff $\alpha=\varepsilon$. By induction on $i$ it is easy to show that $\alpha \sim_{i} \alpha^{\prime}$ implies $\alpha \beta \sim_{i} \alpha^{\prime} \beta$ and $\beta \alpha \sim_{i} \beta \alpha^{\prime}$.

(5) By (4) and (1), $\alpha \sim_{i} \gamma \alpha$ implies $\gamma \alpha \sim_{i} \gamma \gamma \alpha, \gamma \gamma \alpha \sim_{i} \gamma \gamma \gamma \alpha, \ldots$, and thus also $\alpha \sim_{i} \gamma^{i} \alpha$. The obvious fact $\gamma^{i} \alpha \sim_{i} \gamma^{\omega}$ (when $\gamma \neq \varepsilon$ ) thus establishes the claim.

Remark. The "no dead nonterminal" assumption is not crucial for the problem BPABIsIm, since we can always add a special nonterminal $D$ and a special action $d$, with the rules $A \stackrel{d}{\longrightarrow} A$ for all dead nonterminals $A$ (including $D$ ), and finally replace the question $X \stackrel{?}{\sim} Y$ with $X D \stackrel{?}{\sim} Y D$.

Points (1)-(3) in Prop. 3.1 suggest to define the equivalence level, or the eq-level, for each pair of strings:

$$
\operatorname{EQLv}(\alpha, \beta)=k \in \mathbb{N} \text { if } \alpha \sim_{k} \beta \text { and } \alpha \not_{k+1} \beta \text {, and } \operatorname{EQLv}(\alpha, \beta)=\omega \text { if } \alpha \sim \beta \text {. }
$$

We stipulate $n<\omega$ and $\omega+n=\omega-n=\omega+\omega=\omega$ for each $n \in \mathbb{N}$.

We observe the following facts.

\section{Proposition 3.2.}

(1) If $\operatorname{EQLV}(\alpha, \beta)<\omega$ then either there is a transition $\alpha \stackrel{a}{\longrightarrow} \alpha^{\prime}$ such that for any $\beta \stackrel{a}{\longrightarrow} \beta^{\prime}$ we have $\operatorname{EQLV}\left(\alpha^{\prime}, \beta^{\prime}\right)<\operatorname{EQLv}(\alpha, \beta)$, or there is a transition $\beta \stackrel{a}{\longrightarrow} \beta^{\prime}$ such that for any $\alpha \stackrel{a}{\longrightarrow} \alpha^{\prime}$ we have $\operatorname{EQLv}\left(\alpha^{\prime}, \beta^{\prime}\right)<\operatorname{EQLv}(\alpha, \beta)$.

(2) If $\alpha \stackrel{a_{1}}{\longrightarrow} \alpha_{1} \stackrel{a_{2}}{\longrightarrow} \alpha_{2} \cdots \stackrel{a_{k}}{\longrightarrow} \alpha_{k}$ where $a_{i} \in \mathcal{A}$ (for all $i, 1 \leq i \leq k$ ) and $k \leq \operatorname{EQLv}(\alpha, \beta)$ then there are $\beta_{1}, \beta_{2}, \ldots, \beta_{k}$ such that $\beta \stackrel{a_{1}}{\longrightarrow} \beta_{1} \stackrel{a_{2}}{\longrightarrow} \beta_{2} \cdots \stackrel{a_{k}}{\longrightarrow} \beta_{k}$ and

$\operatorname{EQLv}\left(\alpha_{i}, \beta_{i}\right) \geq \operatorname{EQLv}(\alpha, \beta)-i$ for $i=1,2, \ldots, k$; this implies $\alpha_{i} \sim \beta_{i}$ if $\alpha \sim \beta$.

(3) If $\operatorname{EQLv}\left(\alpha, \alpha^{\prime}\right) \geq \operatorname{EQLv}(\alpha, \beta)+1$ then $\operatorname{EQLv}(\alpha, \beta)=\operatorname{EQLv}\left(\alpha^{\prime}, \beta\right)$.

(4) $\operatorname{EQLv}(\alpha, \beta) \leq \operatorname{EQLv}(\alpha \gamma, \beta \gamma)$.

Proof. The claims easily follow from the definitions of $\sim_{i}$ and $\sim$. In Point 2 we can use induction on $k$. For Point 3 it suffices to note that if $\alpha \sim_{i} \beta, \alpha \chi_{i+1} \beta$, and $\alpha \sim_{i+1} \alpha^{\prime}$ (hence also $\alpha \sim_{i} \alpha^{\prime}$ ) then $\alpha^{\prime} \sim_{i} \beta$ and $\alpha^{\prime} \chi_{i+1} \beta$. For Point 4 we note that $\alpha \sim_{i} \beta$ implies $\alpha \gamma \sim_{i} \beta \gamma$ by Prop 3.1 $(1,4)$.

Now we define the norm as a mapping $\mathcal{S}_{\mathcal{G}} \rightarrow \mathbb{N} \cup\{\omega\}$.

Definition 3.3. The norm of $\alpha \in \mathcal{S}_{\mathcal{G}}$ is denoted by $\|\alpha\|$. If there is no $w \in \mathcal{A}^{*}$ such that $\alpha \stackrel{w}{\longrightarrow} \varepsilon$ then we put $\|\alpha\|=\omega$ and say that $\alpha$ is unnormed; otherwise $\alpha$ is normed and $\|\alpha\|=|w|$ for a shortest $w$ such that $\alpha \stackrel{w}{\longrightarrow} \varepsilon$.

A path $\beta_{0} \stackrel{a_{1}}{\longrightarrow} \beta_{1} \stackrel{a_{2}}{\longrightarrow} \beta_{2} \cdots \stackrel{a_{k}}{\longrightarrow} \beta_{k}$ in $\mathcal{L}_{\mathcal{G}}$, where $k \geq 1$ and $a_{i} \in \mathcal{A}$, is norm-reducing if $\left\|\beta_{i}\right\|>\left\|\beta_{i+1}\right\|$ (and thus necessarily $\left\|\beta_{i+1}\right\|=\left\|\beta_{i}\right\|-1$ ) for $i=0,1, \ldots, k-1$.

We note that $\|\varepsilon\|=0$ and $\|\alpha \beta\|=\|\alpha\|+\|\beta\|$. We have $\|\alpha\|=\omega$ when $\alpha$ is infinite. Now we observe further simple facts.

\section{Proposition 3.4.}

(1) If $\|\alpha\| \neq\|\beta\|$ then $\operatorname{EQLv}(\alpha, \beta) \leq \min \{\|\alpha\|,\|\beta\|\}$ (and thus $\alpha \nsim \beta$ ).

(2) If $U \in \mathcal{N}$ and $\|U\|=\omega$ then $U \sim U \alpha$ for any $\alpha$.

(3) $\operatorname{EQLv}(\gamma \alpha, \gamma \beta) \geq\|\gamma\|+\operatorname{EQLv}(\alpha, \beta)$. 
Proof. (1) Suppose $\|\alpha\|<\|\beta\|$. Hence $\alpha \stackrel{u}{\longrightarrow} \varepsilon$ for some $u$ where $|u|=\|\alpha\|$. If $\operatorname{EQLv}(\alpha, \beta) \geq$ $\|\alpha\|$ then there is $\beta^{\prime}$ such that $\beta \stackrel{u}{\longrightarrow} \beta^{\prime}$ and $\operatorname{EQLv}\left(\varepsilon, \beta^{\prime}\right) \geq \operatorname{EQLv}(\alpha, \beta)-\|\alpha\|$ (by Prop. 3.2(2)). Since $\|\beta\|>\|\alpha\|$, we have $\beta^{\prime} \neq \varepsilon$, and thus $\operatorname{EQLv}\left(\varepsilon, \beta^{\prime}\right)=0$. Hence $\operatorname{EQLv}(\alpha, \beta) \leq\|\alpha\|$.

(2) We can easily check that the set $\{(\alpha \gamma, \beta \delta) \mid \alpha \sim \beta,\|\alpha\|=\|\beta\|=\omega\}$ is a bisimulation.

(3) If $\gamma \alpha \sim \gamma \beta$ (which surely holds when $\|\gamma\|=\omega$ ) then the claim is trivial. We thus assume $\gamma \alpha \nsim \gamma \beta$ and proceed by induction on $\operatorname{EQLV}(\gamma \alpha, \gamma \beta)$. If $\operatorname{EQLv}(\gamma \alpha, \gamma \beta)=0$ then $\gamma=\varepsilon$ (hence $\|\gamma\|=0$ ) and the claim is trivial. If $\gamma \neq \varepsilon$ then Prop. 3.2(1) implies that there is a transition $\gamma \stackrel{a}{\longrightarrow} \sigma$, where necessarily $\|\sigma\| \geq\|\gamma\|-1$, such that $\operatorname{EQLv}(\sigma \alpha, \sigma \beta)<$ $\operatorname{EQLv}(\gamma \alpha, \gamma \beta)$. Since $\operatorname{EQLv}(\sigma \alpha, \sigma \beta) \geq\|\sigma\|+\operatorname{EQLv}(\alpha, \beta)$ by the induction hypothesis, we deduce $\operatorname{EQLv}(\gamma \alpha, \gamma \beta) \geq 1+\|\sigma\|+\operatorname{EQLv}(\alpha, \beta) \geq\|\gamma\|+\operatorname{EQLv}(\alpha, \beta)$.

Convention. Prop. 3.4(2) allows us to remove the suffix after the first occurrence of an unnormed nonterminal in any string, without changing its bisimulation equivalence class. We thus further implicitly assume that the considered strings are of the forms $\alpha, \alpha U$, or $\beta \gamma^{\omega}$ where $\alpha, \beta, \gamma \in \mathcal{N}^{*}$ are normed and $U \in \mathcal{N}$ is unnormed. We still might write, e.g., $\gamma \beta$ or $\gamma^{\omega}$ even if $\|\gamma\|=\omega$ but such strings are implicitly identified with the appropriate prefix of $\gamma$.

It will be useful to use the norm when measuring the size of string presentations:

Definition 3.5. Given $\mathcal{G}=(\mathcal{N}, \mathcal{A}, \mathcal{R})$, the function sIZE : $\mathcal{S}_{\mathcal{G}} \cup\left(\mathcal{S}_{\mathcal{G}} \times \mathcal{S}_{\mathcal{G}}\right) \rightarrow \mathbb{N}$ is defined as follows.

- For a finite string $\alpha$ we put $\operatorname{sizE}(\alpha)=\left\|\alpha^{\prime}\right\|$ where $\alpha^{\prime}$ is the longest normed prefix of $\alpha$. (Thus $\operatorname{SizE}(\alpha U)=\operatorname{SizE}(\alpha)=\|\alpha\|$ when $\alpha$ is normed and $U$ is unnormed.)

- For an infinite regular string $\alpha$, containing no unnormed nonterminal, we put $\operatorname{sizE}(\alpha)=$ $\left\|\alpha_{p} \alpha_{c}\right\|$ (where $\alpha_{p}\left(\alpha_{c}\right)^{\omega}$ is the canonical presentation of $\alpha$ ).

- For a pair $(\alpha, \beta)$ we put $\operatorname{sizE}(\alpha, \beta)=\max \{\operatorname{sizE}(\alpha), \operatorname{sizE}(\beta)\}$.

Stipulating $\max \emptyset=0$, we define:

$$
\begin{aligned}
& M=\max \{\|A\| ; A \in \mathcal{N},\|A\|<\omega\}, \\
& M_{r h s}=\max \{\|\alpha\| ;\|\alpha\|<\omega \text { and } \mathcal{R} \text { contains a rule } A \stackrel{a}{\longrightarrow} \alpha\}, \\
& S_{r h s}=\max \{\operatorname{sizE}(\alpha) \mid \mathcal{R} \text { contains a rule } A \stackrel{a}{\longrightarrow} \alpha\} .
\end{aligned}
$$

Hence $M$ is the maximal norm of normed nonterminals, and $S_{r h s}$ is the maximal size of the right-hand sides (rhs) in the rules of $\mathcal{G}$; in particular, $S_{r h s}$ is greater than or equal to the norm of any normed rhs, and thus $M_{r h s} \leq S_{r h s}$.

The following fact is also standard; we sketch a proof to be self-contained.

Proposition 3.6. There is a polynomial-time algorithm which, given $\mathcal{G}=(\mathcal{N}, \mathcal{A}, \mathcal{R})$, computes $\|A\|$ for each $A \in \mathcal{N}$, and also $M, M_{r h s}, S_{r h s}$; these values are bounded by an exponential function of the size of $\mathcal{G}$.

Proof. We sketch an algorithm which outputs nonterminals in an order $A_{1}, A_{2}, \ldots, A_{k}$ (for $k=\operatorname{card}(\mathcal{N}))$ where $\left\|A_{1}\right\| \leq\left\|A_{2}\right\| \leq \cdots \leq\left\|A_{k}\right\|$. Suppose $A_{1}, A_{2}, \ldots, A_{i}$ and their norms have been already established ( $i=0$ in the beginning). Construct the set

$$
\mathcal{D}=\left\{\alpha \mid \alpha \in\left\{A_{1}, A_{2}, \ldots, A_{i}\right\}^{*} \text { and there is a rule } A \stackrel{a}{\longrightarrow} \alpha \text { for } A \notin\left\{A_{1}, A_{2}, \ldots, A_{i}\right\}\right\} .
$$

If $\mathcal{D} \neq \emptyset$ then put $m=\min \{\|\alpha\| ; \alpha \in \mathcal{D}\}$ and define $A_{i+1}$ as a chosen $A \notin\left\{A_{1}, A_{2}, \ldots, A_{i}\right\}$ for which there is a rule $A \stackrel{a}{\longrightarrow} \alpha$ such that $\alpha \in \mathcal{D}$ and $\|\alpha\|=m$; it is obvious that $\left\|A_{i+1}\right\|=1+m$. If $\mathcal{D}=\emptyset$ then $\|A\|=\omega$ for all $A \notin\left\{A_{1}, A_{2}, \ldots, A_{i}\right\}$. The time complexity 
of the algorithm is obviously polynomial. The exponential bounds follow by noting that $\left\|A_{i}\right\| \leq M_{i}$ where we put $M_{0}=0$ and $M_{i+1}=1+r \cdot M_{i}$ for $r=\max \{|\alpha| ; \alpha$ is the rhs of a rule in $\mathcal{R}\}$.

Remark. The exponential upper bound in the proof is tight: if we have the rules $A_{k} \stackrel{a}{\longrightarrow} A_{k-1} A_{k-1}, \ldots, A_{i} \stackrel{a}{\longrightarrow} A_{i-1} A_{i-1}, \ldots, A_{2} \stackrel{a}{\longrightarrow} A_{1} A_{1}, A_{1} \stackrel{a}{\longrightarrow} \varepsilon$ then $\left\|A_{i}\right\|=2^{i}-1$.

We now define a crucial notion, used in the later Prover-Refuter game.

Definition 3.7. A nonempty set $\left\{\left(\alpha_{1}, \beta_{1}\right),\left(\alpha_{2}, \beta_{2}\right), \ldots,\left(\alpha_{k}, \beta_{k}\right)\right\}$ is a decomposition of $(\alpha, \beta)$ if $\operatorname{sizE}\left(\alpha_{j}, \beta_{j}\right)<\operatorname{sizE}(\alpha, \beta)$ for $j=1,2, \ldots, k$, and $(\alpha, \beta)$ belongs to the least congruence (w.r.t. concatenation) containing all $\left(\alpha_{j}, \beta_{j}\right), j=1,2, \ldots, k$. Moreover, if $\alpha_{j} \sim \beta_{j}$ for all $j=1,2, \ldots, k$ then it is a bisimilar decomposition.

Example 3.8. One decomposition of $(A \alpha, B \beta)$ is $\{(A \gamma, B),(\alpha, \gamma \beta)\}$ when both $\operatorname{sizE}(A \gamma, B)$ and $\operatorname{SIZE}(\alpha, \gamma \beta)$ are less than $\operatorname{SIZE}(A \alpha, B \beta)$. Indeed, a least congruence proof is the sequence $(A \gamma, B),(\alpha, \gamma \beta),(\beta, \beta),(A \gamma \beta, B \beta),(A, A),(A \alpha, A \gamma \beta),(A \alpha, B \beta)$ where each pair either is a generator $((A \gamma, B)$ or $(\alpha, \gamma \beta)$ in our case) or is deduced from the previous pairs by using reflexivity, symmetry, transitivity, and concatenation. Another decomposition of $(A \alpha, B \beta)$ is $\left\{(\alpha, \gamma \beta),\left(\beta, \delta^{\omega}\right),\left(A \gamma \delta^{\omega}, B \delta^{\omega}\right)\right\}$ if the size conditions are satisfied.

Proposition 3.9. If $\left\{\left(\alpha_{j}, \beta_{j}\right) \mid 1 \leq j \leq k\right\}$ is a decomposition of $(\alpha, \beta)$ then

$$
\min \left\{\operatorname{EQLv}\left(\alpha_{j}, \beta_{j}\right) \mid 1 \leq j \leq k\right\} \leq \operatorname{EQLv}(\alpha, \beta) ;
$$

if it is a bisimilar decomposition then $\alpha \sim \beta$.

Proof. Let $(\alpha, \beta)$ belong to the least congruence generated by $\left\{\left(\alpha_{j}, \beta_{j}\right) \mid 1 \leq j \leq k\right\}$. Then there is a least congruence proof $\left(\gamma_{1}, \delta_{1}\right),\left(\gamma_{2}, \delta_{2}\right), \ldots,\left(\gamma_{m}, \delta_{m}\right)$ such that $\left(\gamma_{m}, \delta_{m}\right)=$ $(\alpha, \beta)$, and $\left(\gamma_{i}, \delta_{i}\right)$, for each $i, 1 \leq i \leq m$, either is a generator $\left(\alpha_{j}, \beta_{j}\right)$, or satisfies $\gamma_{i}=$ $\delta_{i}$ (reflexivity), or can be derived from pairs $\left(\gamma_{1}, \delta_{1}\right),\left(\gamma_{2}, \delta_{2}\right), \ldots,\left(\gamma_{i-1}, \delta_{i-1}\right)$ by using symmetry, transitivity, or concatenation $\left(\gamma_{i}=\gamma_{i_{1}} \gamma_{i_{2}}, \delta_{i}=\delta_{i_{1}} \delta_{i_{2}}\right.$ for some $\left.i_{1}<i, i_{2}<i\right)$.

For any $\ell \in \mathbb{N}$, by using the fact that $\sim_{\ell}$ is a congruence w.r.t. concatenation (as follows from Prop. 3.1(1,4)) we get: if $\alpha_{j} \sim_{\ell} \beta_{j}$ for all $j, 1 \leq j \leq k$, then $\gamma_{i} \sim_{\ell} \delta_{i}$ for $i=1,2, \ldots, m$, and thus $\alpha \sim_{\ell} \beta$. Hence if $\alpha_{j} \sim_{\ell} \beta_{j}$ for all $j, 1 \leq j \leq k$, and all $\ell \in \mathbb{N}$ then $\alpha_{\ell} \sim_{\ell} \beta$ for all $\ell \in \mathbb{N}$, and thus $\alpha \sim \beta$ (by Prop. 3.1.1(3)).

3.2. Algorithm deciding BPA-Bisim, based on a Prover-Refuter game. We recall that 2-EXPTIME =AEXPSPACE where "A" stands for "Alternating" 7]. For proving Theorem 2.5 it is thus sufficient to show an alternating Turing machine working in exponential space which accepts precisely those $\mathcal{G}, X, Y$ where $X \nsim Y$. The existence of such a machine easily follows from the following game, once we show that Refuter has a winning strategy iff $X \nsim Y$.

Prover (she) - Refuter (he) Game

(1) A BPA-system $\mathcal{G}=(\mathcal{N}, \mathcal{A}, \mathcal{R})$ and $X, Y \in \mathcal{N}$ are given.

(2) A work space of size $2^{\text {pol }(\text { size }(\mathcal{G}))}$ is reserved, where pol is a (sufficiently large) polynomial whose existence will become clear later. A part of the work space serves for storing a presentation of a current pair, initially $(X, Y)$; the rest of the work space is called the free work space.

(3) For $i=1,2,3, \ldots$, the following Phase $i$ is performed; $(\alpha, \beta)$ denotes the current pair: 
(a) If $\alpha \chi_{1} \beta$ then Refuter wins. If $\alpha, \beta$ are dead (i.e., if they do not enable any action, i.e. $\alpha=\beta=\varepsilon$ ) then Prover wins. The play finishes in these cases; otherwise it continues with (b).

(b) Prover can decide to show some (freely chosen) pairs and demonstrate that these pairs constitute a decomposition of $(\alpha, \beta)$. She is restricted by the free work space when presenting the pairs and a least congruence proof. (As shown later, it suffices to allow only decompositions with at most three pairs.) Then Refuter chooses a pair $\left(\alpha^{\prime}, \beta^{\prime}\right)$ from the decomposition and replaces the current pair $(\alpha, \beta)$ with $\left(\alpha^{\prime}, \beta^{\prime}\right)$. (Recall that $\operatorname{SizE}\left(\alpha^{\prime}, \beta^{\prime}\right)<\operatorname{SIZE}(\alpha, \beta)$.) The play then continues with Phase $i+1$.

(c) (Prover has not used the possibility in (b).) Refuter chooses a transition $\alpha \stackrel{a}{\longrightarrow} \alpha^{\prime}$ or $\beta \stackrel{a}{\longrightarrow} \beta^{\prime}$. In the first case Prover chooses some $\beta \stackrel{a}{\longrightarrow} \beta^{\prime}$, in the second case Prover chooses some $\alpha \stackrel{a}{\longrightarrow} \alpha^{\prime}$. If $\left(\alpha^{\prime}, \beta^{\prime}\right)$ does not fit into the space reserved for the current pair then Refuter wins; otherwise the current pair $(\alpha, \beta)$ is replaced with $\left(\alpha^{\prime}, \beta^{\prime}\right)$ and the play continues with Phase $i+1$.

Remark. A play can be infinite, which can be viewed as a win of Prover. To make each play finite, we could add a step counter whose overflow (over a double exponential bound) would mean that a game configuration has been repeated and that Prover has won, but this is not technically necessary.

Lemma 3.10. (Soundness.) If $X \nsim Y$ then Refuter has a winning strategy (even in the game with no space restriction).

Proof. Assume that $X \nsim Y$ and Refuter uses the following strategy. In (b) he always chooses a pair $\left(\alpha^{\prime}, \beta^{\prime}\right)$ with the least eq-level, and in (c) he always chooses a transition guaranteeing that $\operatorname{EQLv}\left(\alpha^{\prime}, \beta^{\prime}\right)<\operatorname{EQLv}(\alpha, \beta)$. Prop. 3.2(1) and Prop. 3.9 show that this is possible and that $\operatorname{EQLv}\left(\alpha^{\prime}, \beta^{\prime}\right)<\operatorname{EQLv}(\alpha, \beta)$, or $\operatorname{EQLv}\left(\alpha^{\prime}, \beta^{\prime}\right)=\operatorname{EQLv}(\alpha, \beta)$ and $\operatorname{size}\left(\alpha^{\prime}, \beta^{\prime}\right)<$ $\operatorname{SIZE}(\alpha, \beta)$. Refuter thus must win eventually; he can only benefit from any space restriction.

In the next subsection we show the completeness (Prover has a winning strategy when $X \sim Y$ ) by which a proof of Theorem 2.5 will be finished.

3.3. Completeness of the Prover-Refuter game. Our aim is to prove Lemma 3.15, a crucial technical fact is captured by the next lemma (assuming a given $\mathcal{G}=(\mathcal{N}, \mathcal{A}, \mathcal{R})$ ):

Lemma 3.11. If $\alpha_{1} \not \alpha_{2}$ and $\alpha_{1} \beta \sim \alpha_{2} \beta$ then there is $\delta \neq \varepsilon$ such that $\beta \sim \delta \beta$ (and thus $\left.\beta \sim \delta^{\omega}\right)$ and $\operatorname{sizE}(\delta) \leq\left(\operatorname{sizE}\left(\alpha_{1}, \alpha_{2}\right)+\operatorname{card}(\mathcal{N})^{2} \cdot M_{r h s}+S_{r h s}\right) \cdot\left(1+S_{r h s}\right)$.

In the lemma we can have $\|\delta\|=\omega$; in this case $\delta \beta=\delta^{\omega}=\delta$ (by our convention after Prop. (3.4). We postpone a proof of this lemma, and a related discussion of normed BPA, to Subsection 3.4 and Section 4. Now we observe a bound on the possible increase of the string size in any transition in $\mathcal{L}_{\mathcal{G}}$. Roughly speaking, by performing a transition the canonical cycle either does not change, or is swapped, or becomes empty; the canonical prefix can increase by $S_{r h s}$ at most.

Proposition 3.12. If $\alpha \stackrel{a}{\longrightarrow} \delta$, i.e. $\alpha_{p}\left(\alpha_{c}\right)^{\omega} \stackrel{a}{\longrightarrow} \delta_{p}\left(\delta_{c}\right)^{\omega}$, then $\delta_{c} \in \operatorname{SWAP}\left(\alpha_{c}\right)$ or $\delta_{c}=\varepsilon$, hence $\operatorname{SIZE}\left(\delta_{c}\right) \leq \operatorname{SIZE}\left(\alpha_{c}\right)$, and $\operatorname{SIZE}\left(\delta_{p}\right) \leq \operatorname{SIZE}\left(\alpha_{p}\right)+S_{r h s}$.

Proof. We have $\alpha \stackrel{a}{\longrightarrow} \delta$ due to a rule $A \stackrel{a}{\longrightarrow} \gamma$, where $\alpha=A \alpha^{\prime}$ and $\delta=\gamma \alpha^{\prime}$. 
If $\|\gamma\|=\omega$ then $\delta=\gamma$ (by Convention after Prop. 3.4), which entails $\delta_{p}=\gamma, \delta_{c}=\varepsilon$, and $\operatorname{SIZE}\left(\delta_{p}\right) \leq S_{r h s}$.

If $\|\gamma\|<\omega$ then (also $\|A\|<\omega$ and) $\delta_{c} \in \operatorname{swaP}\left(\alpha_{c}\right)$ by Prop. 2.3, Recalling Lemma 2.2. we note that if $\alpha_{p} \neq \varepsilon$ then $\alpha_{p}=A \alpha_{p}^{\prime}$, and $\delta_{p}$ is a prefix of $\gamma \alpha_{p}^{\prime}$; this entails $\operatorname{SizE}\left(\delta_{p}\right)<$ $\operatorname{SIZE}\left(\alpha_{p}\right)+S_{r h s}$. If $\alpha_{p}=\varepsilon$ then $\alpha=\left(\alpha_{c}\right)^{\omega}=A \beta^{\omega}$ where $\beta \in \operatorname{sWAP}\left(\alpha_{c}\right)$; hence $\delta=\gamma \beta^{\omega}$, which entails that $\delta_{p}$ is a prefix of $\gamma$ and thus $\operatorname{SIZE}\left(\delta_{p}\right) \leq S_{r h s}$.

The next technical lemma, Lemma 3.14, is related to Point 3(b) in the Prover-Refuter game. It aims to show that if the current pair is $(A \alpha, B \beta)$ where $A \alpha \sim B \beta$ and the presentation size of $(A \alpha, B \beta)$ is bigger than an exponential bound then there is a bisimilar decomposition of $(A \alpha, B \beta)$, with at most three pairs and with a least congruence proof of bounded size.

We handle separately the size of canonical prefixes and the size of canonical cycles. Our convention (after Prop. 3.4) implies $\operatorname{SizE}\left(\alpha_{c}\right)=\left\|\alpha_{c}\right\|<\omega$ (including the case $\alpha_{c}=\varepsilon$ ).

Definition 3.13. Given $\mathcal{G}=(\mathcal{N}, \mathcal{A}, \mathcal{R})$ and $\mathcal{E} \in \mathbb{N}$, we say that a (regular) string $\alpha \in$ $\mathcal{N}^{*} \cup \mathcal{N}^{\omega}$ has an $\mathcal{E}$-bounded cycle if $\operatorname{sizE}\left(\alpha_{c}\right) \leq \mathcal{E}$.

In the next lemma, $\mathcal{E}$ is an exponential bound w.r.t. the size of $\mathcal{G}$ (as follows from Prop. 3.6). The chosen $\mathcal{E}$ and the following analysis are a bit generous, since we prefer technical simplicity to more detailed upper bounds.

Lemma 3.14. Given a BPA system $\mathcal{G}=(\mathcal{N}, \mathcal{A}, \mathcal{R})$, we put

$$
\mathcal{E}=\left(2 M+\operatorname{card}(\mathcal{N})^{2} \cdot M_{r h s}+S_{r h s}\right) \cdot\left(1+S_{r h s}\right) .
$$

If $A \alpha \sim B \beta$, both $A \alpha, B \beta$ have $\mathcal{E}$-bounded cycles, and $\operatorname{sizE}\left((A \alpha)_{p},(B \beta)_{p}\right)>2 M+\mathcal{E}$ then there is a bisimilar decomposition $\left\{\left(\alpha_{1}, \beta_{1}\right),\left(\alpha_{2}, \beta_{2}\right),\left(\alpha_{3}, \beta_{3}\right)\right\}$ of $(A \alpha, B \beta)$ where all $\alpha_{j}, \beta_{j}$ $(1 \leq j \leq 3)$ have $\mathcal{E}$-bounded cycles.

Proof. Let us consider $A \alpha=(A \alpha)_{p}\left((A \alpha)_{c}\right)^{\omega}, B \beta=(B \beta)_{p}\left((B \beta)_{c}\right)^{\omega}$ satisfying the assumption. By our convention, $\alpha=\varepsilon$ if $\|A\|=\omega$ and $\beta=\varepsilon$ if $\|B\|=\omega$; w.l.o.g. we assume $\|A\| \leq\|B\|$.

We recall that $\operatorname{Size}(\alpha, \beta)=\max \{\operatorname{SizE}(\alpha), \operatorname{SizE}(\beta)\}$ (by Def. 3.5) and we now show that

$$
\operatorname{SIZE}(\alpha, \beta)<\operatorname{SIZE}(A \alpha, B \beta) \text {. }
$$

This is not valid in general, $\operatorname{since} \operatorname{sizE}(\alpha)<\operatorname{sizE}(A \alpha)$ if and only if $(A \alpha)_{p} \neq \varepsilon$; if $(A \alpha)_{p}=$ $\varepsilon$ then $A \alpha=\left((A \alpha)_{c}\right)^{\omega}, \alpha=\left((\alpha)_{c}\right)^{\omega}$, and $\alpha_{c} \in \operatorname{swAP}\left((A \alpha)_{c}\right)$, which implies SIZE $(\alpha)=$ $\operatorname{SIZE}(A \alpha)$. In our case we thus have $\operatorname{SizE}(\alpha)<\operatorname{sizE}(A \alpha)$ or $\operatorname{SIZE}(\alpha)=\operatorname{size}(A \alpha) \leq \mathcal{E}$, and $\operatorname{SizE}(\beta)<\operatorname{SizE}(B \beta)$ or $\operatorname{sizE}(\beta)=\operatorname{SIZE}(B \beta) \leq \mathcal{E}$. Since $\operatorname{sizE}\left((A \alpha)_{p},(B \beta)_{p}\right)>2 M+\mathcal{E}$, we indeed easily establish (3.1). Moreover, both $\alpha, \beta$ have $\mathcal{E}$-bounded cycles as well.

Now we perform a case analysis (showing also some decompositions with even less than three pairs); recall that we assume $\|A\| \leq\|B\|$.

(1) $\|A\| \leq\|B\|=\omega$; hence $\beta=\varepsilon,\|A\|<\omega, \alpha \neq \varepsilon$ (since $\operatorname{size}(A \alpha)>2 M+\mathcal{E}>M$ ), and $A \alpha \sim B:$

There is a norm-reducing path $A \stackrel{u}{\longrightarrow} \varepsilon$, where $|u|=\|A\| \leq M$; we have $A \alpha \stackrel{u}{\longrightarrow} \alpha$. By Prop. $3.2(2)$ there is $\gamma$ such that $B \stackrel{u}{\longrightarrow} \gamma$ and $\alpha \sim \gamma$, and thus also $A \gamma \sim B$ (by Prop. 3.1 $(1,4))$; recalling Prop. 3.12, we derive that $\operatorname{sizE}(\gamma) \leq \operatorname{sizE}(B)+M \cdot S_{r h s}=$ $M \cdot S_{r h s}$.

We easily check that both $\operatorname{SizE}(\alpha, \gamma)$ and $\operatorname{SizE}(A \gamma, B)$ are less than $\operatorname{sizE}(A \alpha, B)$, and that $\{(\alpha, \gamma),(A \gamma, B)\}$ is a bisimilar decomposition of $(A \alpha, B)$ (as shown by the least 
congruence proof $(\alpha, \gamma),(A, A),(A \alpha, A \gamma),(A \gamma, B),(A \alpha, B))$; moreover, all strings in the decomposition have $\mathcal{E}$-bounded cycles (which are empty for $\gamma, A \gamma, B$ ).

(2) $\|A\| \leq\|B\|<\omega$ (and $A \alpha \sim B \beta$ ); we consider the disjoint cases (a) and (b):

(a) There is norm-reducing $B \stackrel{v}{\longrightarrow} \varepsilon$ (hence $|v|=\|B\|$, and $B \beta \stackrel{v}{\longrightarrow} \beta$ ) such that $A \stackrel{v}{\longrightarrow} \delta$ for some $\delta \neq \varepsilon$ where $\delta \alpha \sim \beta$ :

For any norm-reducing $A \stackrel{u}{\longrightarrow} \varepsilon$ there is surely $\gamma$ such that $B \stackrel{u}{\longrightarrow} \gamma$ and $\alpha \sim \gamma \beta$ (since $\|A\| \leq\|B\|$ and $A \alpha \sim B \beta$ ). Since $\operatorname{size}(A)=\|A\| \leq M$ and $\operatorname{size}(B)=$ $\|B\| \leq M$, for (finite) strings $\gamma, \delta$ we get

$$
\operatorname{SIZE}(\gamma) \leq M \cdot\left(1+S_{r h s}\right) \leq \frac{\mathcal{E}}{2}, \operatorname{SIZE}(\delta) \leq M \cdot\left(1+S_{r h s}\right) \leq \frac{\mathcal{E}}{2} .
$$

Since $\alpha \sim \gamma \beta \sim \gamma \delta \alpha \sim(\gamma \delta)^{\omega}$ (recall Prop. 3.1(5)), and similarly $\beta \sim(\delta \gamma)^{\omega}$, the set $\left.\left\{\left(\alpha,(\gamma \delta)^{\omega}\right)\right),\left(\beta,(\delta \gamma)^{\omega}\right),\left(A(\gamma \delta)^{\omega}, B(\delta \gamma)^{\omega}\right)\right\}$ can be easily checked to be a bisimilar decomposition of $(A \alpha, B \beta)$; moreover, all strings in the decomposition have $\mathcal{E}$-bounded cycles. (By our convention $(\delta \gamma)^{\omega}=\delta$ if $\|\delta\|=\omega$, etc.)

(b) The condition (a) does not hold:

Let us consider a norm-reducing path $B \stackrel{a_{1}}{\longrightarrow} \gamma_{1} \stackrel{a_{2}}{\longrightarrow} \gamma_{2} \ldots \stackrel{a_{k}}{\longrightarrow} \gamma_{k}=\varepsilon(k=\|B\|)$, and the corresponding path $B \beta \stackrel{a_{1}}{\longrightarrow} \gamma_{1} \beta \stackrel{a_{2}}{\longrightarrow} \gamma_{2} \beta \cdots \stackrel{a_{k}}{\longrightarrow} \gamma_{k} \beta=\beta$. By Prop. 3.2(2) there is a path $A \alpha \stackrel{a_{1}}{\longrightarrow} \alpha_{1} \stackrel{a_{2}}{\longrightarrow} \alpha_{2} \ldots \stackrel{a_{k}}{\longrightarrow} \alpha_{k}$ such that $\alpha_{j} \sim \gamma_{j} \beta$ for $j=1,2, \ldots, k$. Since (a) does not hold, there must be $i \in\{1,2, \ldots, k\}$ such that $\alpha_{i}=\alpha$ ( $A$ has been erased, and $\alpha$ has been exposed); let us put $\gamma=\gamma_{i}$. We thus have $\alpha \sim \gamma \beta$ where $\|\gamma\|<\|B\| \leq M$.

If $(B \beta)_{p} \neq \varepsilon$ (hence $B$ is the first symbol of the canonical prefix and $\operatorname{sizE}(B \beta)=$ $\|B\|+\operatorname{SIZE}(\beta))$ then $\operatorname{sizE}(\gamma \beta) \leq\|\gamma\|+\operatorname{sizE}(\beta)<\operatorname{sizE}(B \beta)$. If $(B \beta)_{p}=\varepsilon$ (hence $B \beta=\left((B \beta)_{c}\right)^{\omega}$ and $\left.\operatorname{sizE}(B \beta)=\operatorname{SizE}(\beta) \leq \mathcal{E}\right)$ then $\operatorname{sizE}(\gamma \beta) \leq\|\gamma\|+\operatorname{sizE}(\beta)<$ $M+\mathcal{E}$. The assumption $\operatorname{sizE}\left((A \alpha)_{p},(B \beta)_{p}\right)>2 M+\mathcal{E}$ thus implies

$$
\operatorname{SIZE}(\alpha, \gamma \beta)<\operatorname{SIZE}(A \alpha, B \beta) \text {. }
$$

We now explore the following two subcases separately.

(i) $A \gamma \sim B$ :

Here $\{(A \gamma, B),(\alpha, \gamma \beta)\}$ is a bisimilar decomposition of $(A \alpha, B \beta)$ (we recall Example 3.8), where all strings have $\mathcal{E}$-bounded cycles.

(ii) $A \gamma \nsim B$ (but $A \gamma \beta \sim B \beta$, since $A \alpha \sim B \beta$ and $\alpha \sim \gamma \beta$ ):

Here we use Lemma 3.11, by putting there $\alpha_{1}=A \gamma, \alpha_{2}=B$ we get $\beta \sim \delta^{\omega}$ where $\operatorname{SIZE}(\delta) \leq\left(2 M+\operatorname{card}(\mathcal{N})^{2} \cdot M_{r h s}+S_{r h s}\right) \cdot\left(1+S_{r h s}\right)=\mathcal{E}$. Hence $\left\{(\alpha, \gamma \beta),\left(\beta, \delta^{\omega}\right),\left(A \gamma \delta^{\omega}, B \delta^{\omega}\right)\right\}$ is a bisimilar decomposition of $(A \alpha, B \beta)$, where all strings have $\mathcal{E}$-bounded cycles; since $\operatorname{sizE}\left(A \gamma \delta^{\omega}, B \delta^{\omega}\right) \leq 2 M+\mathcal{E}<$ $\operatorname{SIZE}(A \alpha, B \beta)$, the size conditions indeed hold.

Lemma 3.15. (Completeness.) There is a polynomial pol, used in Point 2 of the ProverRefuter game, such that $X \sim Y$ implies that Prover has a strategy avoiding Refuter's win (the play may be infinite).

Proof. Starting with $X \sim Y$, we let Prover maintain bisimilarity of (the strings in) each current pair. In Point 3(b) of the game Prover only uses bisimilar decompositions of the form presented in the case analysis in the proof of Lemma 3.14, whenever the canonical prefix of a string in the current pair is bigger than $2 M+\mathcal{E}$. Doing this, Prover keeps the property that the strings in any current pair have $\mathcal{E}$-bounded cycles. In Point 3(c) Prover always chooses so that the next current pair is again bisimilar; Prop. 3.12 implies that the $\mathcal{E}$-boundedness of the cycles is kept. 
Adhering to the above strategy, Prover maintains the property that the current pair fits into space $2 \cdot\left(2 \cdot M+2 \cdot \mathcal{E}+S_{r h s}\right)$. The case analysis in the proof of Lemma 3.14 also makes clear that the space $d \cdot \mathcal{E}$, for a fixed (small) constant $d \in \mathbb{N}$ independent of $\mathcal{G}, X, Y$, is sufficient for presenting the appropriate decompositions together with the least congruence proofs. The claim of the lemma thus easily follows.

3.4. Proof of Lemma 3.11. We now prove Lemma 3.11, by which a proof of Theorem 2.5 will be finished. We assume a BPA system $\mathcal{G}=(\mathcal{N}, \mathcal{A}, \mathcal{R})$, with the associated labelled transition system $\mathcal{L}_{\mathcal{G}}=\left(\mathcal{S}_{\mathcal{G}}, \mathcal{A},(\stackrel{a}{\longrightarrow})_{a \in \mathcal{A}}\right)$ and with the values $M, M_{r h s}, S_{r h s}$ (recall Def. 3.5 and Prop. 3.6). The assumed $\mathcal{G}$ is general, the special case of normed BPA systems is discussed in the next section. We first note the following simple fact.

Proposition 3.16. If $\sigma \beta \sim \sigma^{\prime} \beta$ and $\|\sigma\|<\left\|\sigma^{\prime}\right\|$ then there is $\delta \neq \varepsilon$ such that $\beta \sim \delta \beta$ and

$$
\operatorname{SIZE}(\delta) \leq \operatorname{SIZE}\left(\sigma, \sigma^{\prime}\right) \cdot\left(1+S_{r h s}\right) .
$$

Proof. Suppose $\sigma \beta \sim \sigma^{\prime} \beta$ and $\|\sigma\|<\left\|\sigma^{\prime}\right\|$; let $\sigma \stackrel{v}{\longrightarrow} \varepsilon$ be a norm-reducing path. The path $\sigma \beta \stackrel{v}{\longrightarrow} \beta$ must have a matching path $\sigma^{\prime} \beta \stackrel{v}{\longrightarrow} \tau$ such that $\beta \sim \tau$ (recall Prop. [3.2(2)). Since $\left\|\sigma^{\prime}\right\|>\|\sigma\|$, we can write $\tau=\delta \beta$ where $\sigma^{\prime} \stackrel{v}{\longrightarrow} \delta$ and $\delta \neq \varepsilon$; we note that $\operatorname{SizE}(\delta) \leq$ $\operatorname{SIZE}\left(\sigma^{\prime}\right)+|v| \cdot S_{\text {rhs }}$ (using Prop. 3.12 generously). Since $|v|=\|\sigma\| \leq \operatorname{SizE}\left(\sigma, \sigma^{\prime}\right)$, we get

$$
\operatorname{SIZE}(\delta) \leq \operatorname{SIZE}\left(\sigma, \sigma^{\prime}\right)+\operatorname{SIZE}\left(\sigma, \sigma^{\prime}\right) \cdot S_{r h s}=\operatorname{SIZE}\left(\sigma, \sigma^{\prime}\right) \cdot\left(1+S_{r h s}\right) .
$$

Lemma 3.11. (Repeated.) If $\alpha_{1} \nsim \alpha_{2}$ and $\alpha_{1} \beta \sim \alpha_{2} \beta$ then there is $\delta \neq \varepsilon$ such that $\beta \sim \delta \beta$ (and thus $\left.\beta \sim \delta^{\omega}\right)$ and $\operatorname{sizE}(\delta) \leq\left(\operatorname{sizE}\left(\alpha_{1}, \alpha_{2}\right)+\operatorname{card}(\mathcal{N})^{2} \cdot M_{r h s}+S_{r h s}\right) \cdot\left(1+S_{r h s}\right)$.

Proof. In the assumed BPA system $\mathcal{G}=(\mathcal{N}, \mathcal{A}, \mathcal{R})$, for each pair $\left(A_{1}, A_{2}\right)$ of nonterminals where $\left\|A_{1}\right\| \leq\left\|A_{2}\right\|<\omega$ we fix a norm-reducing path $A_{2} \stackrel{u}{\longrightarrow} \gamma$ such that $\|\gamma\|=\left\|A_{2}\right\|-\left\|A_{1}\right\|$ (hence $|u|=\left\|A_{1}\right\|$ ).

Now we consider $\alpha_{1}, \alpha_{2}, \beta$ such that $\alpha_{1} \not \alpha_{2}$ and $\alpha_{1} \beta \sim \alpha_{2} \beta$. At least one of $\alpha_{1}, \alpha_{2}$ must be normed (otherwise $\alpha_{1} \beta \sim \alpha_{1}$ and $\alpha_{2} \beta \sim \alpha_{2}$ ), and we thus have $\left\|\alpha_{1}\right\| \neq\left\|\alpha_{2}\right\|$ or $\left\|\alpha_{1}\right\|=\left\|\alpha_{2}\right\|<\omega$. If $\left\|\alpha_{1}\right\| \neq\left\|\alpha_{2}\right\|$ then the claim of the lemma is true by Prop. 3.16. We thus assume $\left\|\alpha_{1}\right\|=\left\|\alpha_{2}\right\|<\omega$, and imagine a stepwise (not necessarily effective) construction of a certain sequence

$$
\left(\rho_{1}, \rho_{1}^{\prime}, \mu_{1}\right),\left(\rho_{2}, \rho_{2}^{\prime}, \mu_{2}\right), \ldots,\left(\rho_{m}, \rho_{m}^{\prime}, \mu_{m}\right)
$$

where $\left(\rho_{1}, \rho_{1}^{\prime}, \mu_{1}\right)=\left(\alpha_{1}, \alpha_{2}, \varepsilon\right)$. The construction will guarantee that for all $i \in\{1,2, \ldots, m\}$ we have $\rho_{i} \not \rho_{i}^{\prime}, \mu_{i}$ is normed, and $\rho_{i} \mu_{i} \beta \sim \rho_{i}^{\prime} \mu_{i} \beta$; for $i=1$ this holds by the assumptions. Moreover, we will have $\left\|\rho_{i}\right\|=\left\|\rho_{i}^{\prime}\right\|<\omega$ for $i=1,2, \ldots, m-1$, and $\left\|\rho_{m}\right\| \neq\left\|\rho_{m}^{\prime}\right\|$.

Suppose we have constructed $\left(\rho_{i}, \rho_{i}^{\prime}, \mu_{i}\right)$ where $\left\|\rho_{i}\right\|=\left\|\rho_{i}^{\prime}\right\|<\omega, \rho_{i} \not \rho_{i}^{\prime}$, and $\rho_{i} \mu_{i} \beta \sim$ $\rho_{i}^{\prime} \mu_{i} \beta$. Since both $\rho_{i}, \rho_{i}^{\prime}$ are thus nonempty, we can write

$$
\rho_{i}=A_{1} \delta_{1}, \rho_{i}^{\prime}=A_{2} \delta_{2}
$$

where $A_{1}, A_{2} \in \mathcal{N}$ (and $\left\|A_{1} \delta_{1}\right\|=\left\|A_{2} \delta_{2}\right\|<\omega$ ). We assume $\left\|A_{1}\right\| \leq\left\|A_{2}\right\|$ (otherwise we just swap $\left.\rho_{i}, \rho_{i}^{\prime}\right)$; let $A_{2} \stackrel{u}{\longrightarrow} \gamma$ be the norm-reducing path which we fixed for $\left(A_{1}, A_{2}\right)$ above. Recall that $|u|=\left\|A_{1}\right\|,\left\|A_{1} \gamma\right\|=\left\|A_{2}\right\|$ and note that $\left\|\delta_{1}\right\|=\left\|\gamma \delta_{2}\right\|$. We thus have

$$
A_{1} \delta_{1} \nsim A_{2} \delta_{2} \text { and } A_{1} \delta_{1} \mu_{i} \beta \sim A_{2} \delta_{2} \mu_{i} \beta
$$


and we now describe how to choose $\left(\rho_{i+1}, \rho_{i+1}^{\prime}, \mu_{i+1}\right)$, depending on the following cases.

(1) $\left(\rho_{i}, \rho_{i}^{\prime}\right)=\left(A_{1} \gamma, A_{2}\right)$, i.e. $\delta_{1}=\gamma$ and $\delta_{2}=\varepsilon$ in (3.3):

Hence $A_{1} \gamma \not A_{2}$ and $A_{1} \gamma \mu_{i} \beta \sim A_{2} \mu_{i} \beta$. We fix a rule $A_{j} \stackrel{a}{\longrightarrow} \sigma_{j}, j \in\{1,2\}$, such that for any rule $A_{3-j} \stackrel{a}{\longrightarrow} \sigma_{3-j}$ we get $\operatorname{EQLv}\left(A_{1} \gamma, A_{2}\right)>\operatorname{EQLv}\left(\sigma_{1} \gamma, \sigma_{2}\right) ; \operatorname{such} A_{j} \stackrel{a}{\longrightarrow} \sigma_{j}$ exists by Prop. 3.2(1). Now we fix a rule $A_{3-j} \stackrel{a}{\longrightarrow} \sigma_{3-j}$ such that $\sigma_{1} \gamma \mu_{i} \beta \sim \sigma_{2} \mu_{i} \beta$; such $A_{3-j} \stackrel{a}{\longrightarrow} \sigma_{3-j}$ exists by Prop. $\underline{3.2}(2)$. Using the fixed rules $A_{1} \stackrel{a}{\longrightarrow} \sigma_{1}, A_{2} \stackrel{a}{\longrightarrow} \sigma_{2}$, we put

$$
\left(\rho_{i+1}, \rho_{i+1}^{\prime}, \mu_{i+1}\right)=\left(\sigma_{1} \gamma, \sigma_{2}, \mu_{i}\right) .
$$

We note the following properties of our choice:

- $\operatorname{EQLv}\left(\rho_{i+1}, \rho_{i+1}^{\prime}\right)<\operatorname{EQLv}\left(\rho_{i}, \rho_{i}^{\prime}\right)$,

- $\rho_{i+1} \mu_{i+1} \beta \sim \rho_{i+1}^{\prime} \mu_{i+1} \beta$,

- $\min \left\{\left\|\rho_{i+1} \mu_{i+1}\right\|,\left\|\rho_{i+1}^{\prime} \mu_{i+1}\right\|\right\} \leq\left\|\rho_{i} \mu_{i}\right\|+M_{r h s}-1$

(we cannot have $\left\|\sigma_{1}\right\|=\left\|\sigma_{2}\right\|=\omega$ since $\sigma_{1} \gamma \not \sigma_{2}$ and $\sigma_{1} \gamma \mu_{i} \beta \sim \sigma_{2} \mu_{i} \beta$ ),

- $\operatorname{SIZE}\left(\rho_{i+1} \mu_{i+1}, \rho_{i+1}^{\prime} \mu_{i+1}\right) \leq \max \left\{\left\|\rho_{i} \mu_{i}\right\|+M_{r h s}-1, S_{r h s}\right\}$.

We need to count with $S_{r h s}$ since one of $\sigma_{1}, \sigma_{2}$ can be unnormed; in this case one of $\rho_{i+1} \mu_{i+1}, \rho_{i+1}^{\prime} \mu_{i+1}$ is unnormed and its size is at most $S_{r h s}$ (using our convention that $\sigma \tau=\sigma$ when $\|\sigma\|=\omega)$. We have the following two possibilities.

(a) If $\left\|\sigma_{1} \gamma\right\| \neq\left\|\sigma_{2}\right\|$ then $\left\|\rho_{i+1}\right\| \neq\left\|\rho_{i+1}^{\prime}\right\|$ and the sequence (3.2) is completed, i.e. $i+1=m$.

(b) If $\left\|\sigma_{1} \gamma\right\|=\left\|\sigma_{2}\right\|$ then $\left\|\rho_{i+1} \mu_{i+1}\right\|=\left\|\rho_{i+1}^{\prime} \mu_{i+1}\right\|<\omega$.

(2) $\left(\rho_{i}, \rho_{i}^{\prime}\right)=\left(A_{1} \delta_{1}, A_{2} \delta_{2}\right) \neq\left(A_{1} \gamma, A_{2}\right)$, and we have

$$
\operatorname{EQLv}\left(A_{1} \gamma, A_{2}\right) \leq \operatorname{EQLv}\left(A_{1} \delta_{1}, A_{2} \delta_{2}\right) \text { and } A_{1} \gamma \delta_{2} \mu_{i} \beta \sim A_{2} \delta_{2} \mu_{i} \beta:
$$

Here we put

$$
\left(\rho_{i+1}, \rho_{i+1}^{\prime}, \mu_{i+1}\right)=\left(A_{1} \gamma, A_{2}, \delta_{2} \mu_{i}\right)
$$

this choice has the following properties:

- $\operatorname{EQLv}\left(\rho_{i+1}, \rho_{i+1}^{\prime}\right) \leq \operatorname{EQLV}\left(\rho_{i}, \rho_{i}^{\prime}\right)$,

- $\rho_{i+1} \mu_{i+1} \beta \sim \rho_{i+1}^{\prime} \mu_{i+1} \beta$,

- $\left\|\rho_{i+1} \mu_{i+1}\right\|=\left\|\rho_{i+1}^{\prime} \mu_{i+1}\right\|=\left\|\rho_{i} \mu_{i}\right\|=\left\|\rho_{i}^{\prime} \mu_{i}\right\|$.

Moreover, for $i+1$ the above case (1) will apply.

(3) None of (1), (2) applies:

Since (1) and (2) cover precisely the cases where the conjunction (3.5) holds, here we handle the cases where the conjunction (3.5) does not hold. We partition these cases into the disjoint parts (a) and (b) below.

(a) (3.5) does not hold, and $\delta_{1} \mu_{i} \beta \nsim \gamma \delta_{2} \mu_{i} \beta$ :

(The reasoning here is based on the fact $\delta_{1} \mu_{i} \beta \nsim \gamma \delta_{2} \mu_{i} \beta$, and it could be applied even if (3.5) would hold.)

We recall $A_{1} \delta_{1} \mu_{i} \beta \sim A_{2} \delta_{2} \mu_{i} \beta$ from (3.4). Hence the path $A_{2} \delta_{2} \mu_{i} \beta \stackrel{u}{\longrightarrow} \gamma \delta_{2} \mu_{i} \beta$ (corresponding to the fixed norm-reducing path $A_{2} \stackrel{u}{\longrightarrow} \gamma$ ) has a matching path $A_{1} \delta_{1} \mu_{i} \beta \stackrel{u}{\longrightarrow}$ as claimed in Prop. 3.2(2); this path cannot finish in $\delta_{1} \mu_{i} \beta$, since $\delta_{1} \mu_{i} \beta \not \gamma \delta_{2} \mu_{i} \beta$ (i.e., the respective path $A_{1} \stackrel{u}{\longrightarrow}$ cannot be norm-reducing). Though we start with the same norms $\left\|A_{1} \delta_{1}\right\|=\left\|A_{2} \delta_{2}\right\|$, we thus must get a 
difference of norms in the following sense: the path $A_{2} \stackrel{u}{\longrightarrow} \gamma$ has a prefix $A_{2} \stackrel{u_{1}}{\longrightarrow}$ $\sigma_{2} \stackrel{a}{\longrightarrow} \tau_{2}$, where $a \in \mathcal{A}$ (and $u_{1}$ might be empty), such that there is a path $A_{1} \stackrel{u_{1}}{\longrightarrow}$ $\sigma_{1} \stackrel{a}{\longrightarrow} \tau_{1}$ where $\sigma_{1} \delta_{1} \mu_{i} \beta \sim \sigma_{2} \delta_{2} \mu_{i} \beta,\left\|\sigma_{1} \delta_{1}\right\|=\left\|\sigma_{2} \delta_{2}\right\|$, and $\tau_{1} \delta_{1} \mu_{i} \beta \sim \tau_{2} \delta_{2} \mu_{i} \beta$, $\left\|\tau_{1} \delta_{1}\right\|>\left\|\tau_{2} \delta_{2}\right\|$. Here we put

$$
\left(\rho_{i+1}, \rho_{i+1}^{\prime}, \mu_{i+1}\right)=\left(\tau_{1} \delta_{1}, \tau_{2} \delta_{2}, \mu_{i}\right) .
$$

In this case $\left\|\rho_{i+1}\right\| \neq\left\|\rho_{i+1}^{\prime}\right\|$, and (3.2) is completed, i.e. $i+1=m$.

Here we do not claim that $\operatorname{EQLv}\left(\rho_{i+1}, \rho_{i+1}^{\prime}\right) \leq \operatorname{EQLv}\left(\rho_{i}, \rho_{i}^{\prime}\right)$ but we note the following properties:

- $\rho_{i+1} \mu_{i+1} \beta \sim \rho_{i+1}^{\prime} \mu_{i+1} \beta$,

- $\left\|\rho_{i+1} \mu_{i+1}\right\| \neq\left\|\rho_{i+1}^{\prime} \mu_{i+1}\right\|$,

- $\min \left\{\left\|\rho_{i+1} \mu_{i+1}\right\|,\left\|\rho_{i+1}^{\prime} \mu_{i+1}\right\|\right\}=\left\|\rho_{i+1}^{\prime} \mu_{i+1}\right\|<\left\|\rho_{i} \mu_{i}\right\|$,

- $\operatorname{SIZE}\left(\rho_{i+1} \mu_{i+1}\right) \leq \max \left\{\left\|\rho_{i} \mu_{i}\right\|+M_{r h s}-1, S_{r h s}\right\}$.

The last two points follow from the facts that $\left\|\tau_{2} \delta_{2}\right\|<\left\|A_{2} \delta_{2}\right\|$ (since $A_{2} \stackrel{u}{\longrightarrow} \gamma$ is norm-reducing) and that $\tau_{1}$ arises by applying a rule to $\sigma_{1}$; thus $\left\|\tau_{1} \delta_{1}\right\| \leq$ $\left\|\sigma_{1} \delta_{1}\right\|+M_{r h s}-1 \leq\left\|A_{1} \delta_{1}\right\|+M_{r h s}-1$ if $\tau_{1}$ is normed and $\operatorname{sizE}\left(\tau_{1}\right) \leq S_{r h s}$ if $\tau_{1}$ is unnormed (in which case $\rho_{i+1} \mu_{i+1}=\tau_{1}$ ).

(b) (3.5) does not hold, and $\delta_{1} \mu_{i} \beta \sim \gamma \delta_{2} \mu_{i} \beta$ :

We note that $\delta_{1} \mu_{i} \beta \sim \gamma \delta_{2} \mu_{i} \beta$ implies $A_{1} \delta_{1} \mu_{i} \beta \sim A_{1} \gamma \delta_{2} \mu_{i} \beta$, and the assumption $A_{1} \delta_{1} \mu_{i} \beta \sim A_{2} \delta_{2} \mu_{i} \beta$ (3.4) then yields $A_{1} \gamma \delta_{2} \mu_{i} \beta \sim A_{2} \delta_{2} \mu_{i} \beta$; the second conjunct in (3.5) thus holds. Hence the first conjunct does not hold, and we have

$$
\operatorname{EQLv}\left(A_{1} \gamma, A_{2}\right)>\operatorname{EQLv}\left(A_{1} \delta_{1}, A_{2} \delta_{2}\right) \text {. }
$$

We thus have $\operatorname{EQLv}\left(A_{1} \gamma \delta_{2}, A_{2} \delta_{2}\right)>\operatorname{EQLv}\left(A_{1} \delta_{1}, A_{2} \delta_{2}\right)$ (by Prop. $\left.3.2(4)\right)$; this implies that $\operatorname{EQLv}\left(A_{1} \delta_{1}, A_{1} \gamma \delta_{2}\right)=\operatorname{EQLv}\left(A_{1} \delta_{1}, A_{2} \delta_{2}\right)$ (by Prop. 3.2. (3)).

Since $\operatorname{EQLv}\left(A_{1} \delta_{1}, A_{1} \gamma \delta_{2}\right) \geq\left\|A_{1}\right\|+\operatorname{EQLv}\left(\delta_{1}, \gamma \delta_{2}\right)$ (by Prop. 3.4 (3)), we get

We put

$$
\operatorname{EQLv}\left(\delta_{1}, \gamma \delta_{2}\right) \leq \operatorname{EQLv}\left(A_{1} \delta_{1}, A_{1} \gamma \delta_{2}\right)-\left\|A_{1}\right\|=\operatorname{EQLv}\left(A_{1} \delta_{1}, A_{2} \delta_{2}\right)-\left\|A_{1}\right\| .
$$

and note the following properties:

$$
\left(\rho_{i+1}, \rho_{i+1}^{\prime}, \mu_{i+1}\right)=\left(\delta_{1}, \gamma \delta_{2}, \mu_{i}\right)
$$

- $\operatorname{EQLv}\left(\rho_{i+1}, \rho_{i+1}^{\prime}\right) \leq \operatorname{EQLv}\left(\rho_{i}, \rho_{i}^{\prime}\right)-\left\|A_{1}\right\|<\operatorname{EQLv}\left(\rho_{i}, \rho_{i}^{\prime}\right)$,

- $\rho_{i+1} \mu_{i+1} \beta \sim \rho_{i+1}^{\prime} \mu_{i+1} \beta$,

- $\left\|\rho_{i+1} \mu_{i+1}\right\|=\left\|\rho_{i+1}^{\prime} \mu_{i+1}\right\|=\left\|\rho_{i} \mu_{i}\right\|-\left\|A_{1}\right\|$.

If we construct a sequence (3.2) by performing the above described step for $i=1,2,3, \ldots$, we obviously maintain the properties $\rho_{i} \not \rho_{i}^{\prime}$ and $\rho_{i} \mu_{i} \beta \sim \rho_{i}^{\prime} \mu_{i} \beta$. When some $\left(\rho_{i}, \rho_{i}^{\prime}, \mu_{i}\right)$ where $\left\|\rho_{i}\right\| \neq\left\|\rho_{i}^{\prime}\right\|$ is constructed, the construction ends $(i=m$ in (3.2) $)$, and this is the only way how to end. The end is reached whenever the case (3a) applies; another possibility occurs in the case (1). We also maintain that $\mu_{i}$ is normed; $\mu_{i}$ is "increasing" in the sense that $\mu_{i}$ is a suffix of $\mu_{i+1}$ (for $i<m$ ).

Informally speaking, the "head eq-level" is decreasing. More precisely, if (1), (2), or (3b) applies to $i$ then we have $\operatorname{EQLv}\left(\rho_{i}, \rho_{i}^{\prime}\right) \geq \operatorname{EQLv}\left(\rho_{i+1}, \rho_{i+1}^{\prime}\right)$; if (3a) applies then we do not care since the construction finishes (with $i+1=m$ ). In (1) and (3b) the head eq-level is even strictly decreasing, i.e. $\operatorname{EQLv}\left(\rho_{i}, \rho_{i}^{\prime}\right)>\operatorname{EQLv}\left(\rho_{i+1}, \rho_{i+1}^{\prime}\right)$. We thus cannot use (1) for the same pair $\left(A_{1}, A_{2}\right)$ twice; this implies that (1) cannot be used more than $\operatorname{card}(\mathcal{N})^{2}$ times (which is a generous upper bound). Since any use of (2) for $i$ entails using (1) for $i+1$, the head eq-level decreasing guarantees that the construction must end eventually, reaching some $\left(\rho_{m}, \rho_{m}^{\prime}, \mu_{m}\right)$ where $\left\|\rho_{m}\right\| \neq\left\|\rho_{m}^{\prime}\right\|$. 
We recall that $\min \left\{\left\|\rho_{1} \mu_{1}\right\|,\left\|\rho_{1}^{\prime} \mu_{1}\right\|\right\}=\left\|\alpha_{1}\right\|=\left\|\alpha_{2}\right\|<\omega$. We can easily check that for each $i \in\{1,2, \ldots, m-1\}$ we have:

- if (1) applies to $i$ then $\min \left\{\left\|\rho_{i+1} \mu_{i+1}\right\|,\left\|\rho_{i+1}^{\prime} \mu_{i+1}\right\|\right\} \leq \min \left\{\left\|\rho_{i} \mu_{i}\right\|,\left\|\rho_{i}^{\prime} \mu_{i}\right\|\right\}+M_{r h s}$;

- if (2) or (3) applies to $i$ then $\min \left\{\left\|\rho_{i+1} \mu_{i+1}\right\|,\left\|\rho_{i+1}^{\prime} \mu_{i+1}\right\|\right\} \leq \min \left\{\left\|\rho_{i} \mu_{i}\right\|,\left\|\rho_{i}^{\prime} \mu_{i}\right\|\right\}$.

We thus have

$$
\min \left\{\left\|\rho_{m} \mu_{m}\right\|,\left\|\rho_{m}^{\prime} \mu_{m}\right\|\right\} \leq\left\|\alpha_{1}\right\|+\operatorname{card}(\mathcal{N})^{2} \cdot M_{r h s}
$$

If both $\rho_{m}, \rho_{m}^{\prime}$ are normed then

$$
\max \left\{\left\|\rho_{m} \mu_{m}\right\|,\left\|\rho_{m}^{\prime} \mu_{m}\right\|\right\} \leq\left\|\alpha_{1}\right\|+\operatorname{card}(\mathcal{N})^{2} \cdot M_{r h s}+M_{r h s}
$$

in fact, $\max \left\{\left\|\rho_{m} \mu_{m}\right\|,\left\|\rho_{m}^{\prime} \mu_{m}\right\|\right\} \leq \min \left\{\left\|\rho_{m} \mu_{m}\right\|,\left\|\rho_{m}^{\prime} \mu_{m}\right\|\right\}+M_{r h s}$, as can be checked in (1) and (3a). If one of $\rho_{m}, \rho_{m}^{\prime}$ is unnormed then its size is at most $S_{r h s}$. We can thus safely confirm that

$$
\operatorname{SIZE}\left(\rho_{m} \mu_{m}, \rho_{m}^{\prime} \mu_{m}\right) \leq \operatorname{SIZE}\left(\alpha_{1}, \alpha_{2}\right)+\operatorname{card}(\mathcal{N})^{2} \cdot M_{r h s}+S_{r h s} .
$$

Since $\left\|\rho_{m} \mu_{m}\right\| \neq\left\|\rho_{m}^{\prime} \mu_{m}\right\|$ and $\rho_{m} \mu_{m} \beta \sim \rho_{m}^{\prime} \mu_{m} \beta$, Prop. 3.16 finishes the proof.

\section{EXPonential Bound on EQ-LEVELS in NORMEd BPA Systems}

A BPA system is normed if each nonterminal is normed:

Definition 4.1. A $B P A$ system $\mathcal{G}=(\mathcal{N}, \mathcal{A}, \mathcal{R})$ is normed if $\|A\|<\omega$ for all $A \in \mathcal{N}$.

Convention. In this section we stipulate $\mathcal{S}_{\mathcal{G}}=\mathcal{N}^{*}$ in the LTS $\mathcal{L}_{\mathcal{G}}=\left(\mathcal{S}_{\mathcal{G}}, \mathcal{A},(\stackrel{a}{\longrightarrow})_{a \in \mathcal{A}}\right)$; we thus do not consider infinite regular strings (since they are unnormed).

As already mentioned, the problem BPA-BIsIM restricted to normed BPA systems is known to be in PTime. Nevertheless it is easy to construct an example where $\operatorname{EQLv}(X, Y)$ (for $X \not Y$ ) is exponential in the size of the given normed BPA system $\mathcal{G}$; e.g., in Remark after Prop. 3.6 we have $\operatorname{EQLv}\left(A_{k}, A_{k-1}\right)=\left\|A_{k-1}\right\|=2^{k-1}-1$.

An exponential upper bound on the eq-levels in the normed case seems to be only implicit in the literature; we thus show a bound explicitly here, as Theorem4.5. In principle, we use again the construction from the proof of Lemma 3.11 in Subsection 3.4, but now in a different setting and with a different aim. It is easy to note that in the normed case we cannot have $\alpha_{1} \not \alpha_{2}$ and $\alpha_{1} \beta \sim \alpha_{2} \beta$; but this is not a problem, we do not need such $\beta$ here. We will construct a sequence like (3.2), with the decreasing head eqlevels $\operatorname{EQLv}\left(\rho_{i}, \rho_{i}^{\prime}\right)$, but we will now take also the "overall" eq-levels $\operatorname{EQLv}\left(\rho_{i} \mu_{i}, \rho_{i}^{\prime} \mu_{i}\right)$ into account. These eq-levels were of no interest in Subsection 3.4 (there we just took care that $\left.\operatorname{EQLv}\left(\rho_{i} \mu_{i} \beta, \rho_{i}^{\prime} \mu_{i} \beta\right)=\omega\right)$; here these overall eq-levels add technical complications since they can evolve differently than the head eq-levels. We remove these complications when we arrange that $\operatorname{EQLv}\left(\rho_{i} \mu_{i}, \rho_{i}^{\prime} \mu_{i}\right)=\operatorname{EQLv}\left(\rho_{i}, \rho_{i}^{\prime}\right)+\left\|\mu_{i}\right\|$; that's why we introduce the following completion of a normed system with a special unnormed nonterminal.

Definition 4.2. For a normed BPA system $\mathcal{G}=(\mathcal{N}, \mathcal{A}, \mathcal{R})$, by the completion of $\mathcal{G}$ we mean the BPA system $\mathcal{G}^{\prime}=\left(\mathcal{N} \cup\{U\}, \mathcal{A}, \mathcal{R}^{\prime}\right)$ where $U$ is a special (unnormed) nonterminal, and $\mathcal{R}^{\prime}=\mathcal{R} \cup\{U \stackrel{a}{\longrightarrow} U \mid a \in \mathcal{A}\} \cup\{A \stackrel{a}{\longrightarrow} U \mid A \in \mathcal{N}, a \in \mathcal{A}\}$. 
By our conventions, in the LTS $\mathcal{L}_{\mathcal{G}^{\prime}}=\left(\mathcal{S}_{\mathcal{G}^{\prime}}, \mathcal{A},(\stackrel{a}{\longrightarrow})_{a \in \mathcal{A}}\right)$ we have $\mathcal{S}_{\mathcal{G}^{\prime}}=\mathcal{N}^{*} \cup\{\alpha U \mid \alpha \in$ $\left.\mathcal{N}^{*}\right\}$. In $\mathcal{L}_{\mathcal{G}^{\prime}}$ we obviously have $\operatorname{EQLv}(\alpha, \beta)=0$ iff precisely one of $\alpha, \beta$ is $\varepsilon$. Other useful properties of $\mathcal{L}_{\mathcal{G}^{\prime}}$ are captured in Prop. 4.4, but we first make clear that an upper bound on eq-levels in $\mathcal{L}_{\mathcal{G}^{\prime}}$ is also an upper bound on eq-levels in $\mathcal{L}_{\mathcal{G}}$.

\section{Proposition 4.3.}

(1) $\operatorname{EQLv}\left(\gamma_{1}, \gamma_{2}\right)$ in $\mathcal{L}_{\mathcal{G}}$ is not bigger than $\operatorname{EQLv}\left(\gamma_{1}, \gamma_{2}\right)$ in $\mathcal{L}_{\mathcal{G}^{\prime}}$.

(2) In $\mathcal{L}_{\mathcal{G}^{\prime}}$ we have $\alpha \sim U$ iff $\|\alpha\|=\omega$.

(3) For any $\gamma_{1}, \gamma_{2} \in \mathcal{N}^{*}$ we have $\gamma_{1} \sim \gamma_{2}$ in $\mathcal{L}_{\mathcal{G}}$ iff $\gamma_{1} \sim \gamma_{2}$ in $\mathcal{L}_{\mathcal{G}^{\prime}}$.

(Hence if $\operatorname{EQLV}\left(\gamma_{1}, \gamma_{2}\right)$ is finite in $\mathcal{L}_{\mathcal{G}}$ then it is finite in $\mathcal{L}_{\mathcal{G}^{\prime}}$ as well.)

Proof. (1) If $\gamma_{1} \sim_{i} \gamma_{2}$ in $\mathcal{L}_{\mathcal{G}}$ then $\gamma_{1} \sim_{i} \gamma_{2}$ in $\mathcal{L}_{\mathcal{G}^{\prime}}$, as can be shown by induction on $i$, when noting that each move $\gamma_{j} \stackrel{a}{\longrightarrow} U$ can be matched by $\gamma_{3-j} \stackrel{a}{\longrightarrow} U$ if $\gamma_{3-j} \neq \varepsilon$.

(2) If $\|\alpha\|<\|\beta\|$ then $\alpha \nsim \beta$ (recall Prop. 3.4(1)); on the other hand, the set $\{(\alpha, \beta) ;\|\alpha\|=\|\beta\|=\omega\}$ is here a bisimulation.

(3) From Point 1 we get that $\gamma_{1} \sim \gamma_{2}$ in $\mathcal{L}_{\mathcal{G}}$ implies $\gamma_{1} \sim \gamma_{2}$ in $\mathcal{L}_{\mathcal{G}^{\prime}}$; on the other hand, $\left\{(\alpha, \beta) \in \mathcal{N}^{*} \times \mathcal{N}^{*} \mid \alpha \sim \beta\right.$ in $\left.\mathcal{L}_{\mathcal{G}^{\prime}}\right\}$ can be easily checked to be a bisimulation in $\mathcal{L}_{\mathcal{G}}$.

Proposition 4.4. In $\mathcal{L}_{\mathcal{G}^{\prime}}$ the following claims hold:

(1) $\operatorname{EQLv}\left(\gamma_{1}, \gamma_{2}\right)=0$ iff precisely one of $\gamma_{1}, \gamma_{2}$ is the empty word $\varepsilon$.

(2) $\operatorname{EQLv}\left(\gamma_{1}, \gamma_{2}\right) \geq \min \left\{\left\|\gamma_{1}\right\|,\left\|\gamma_{2}\right\|\right\}$.

(3) If $\left\|\gamma_{1}\right\| \neq\left\|\gamma_{2}\right\|$ then $\operatorname{EQLV}\left(\gamma_{1}, \gamma_{2}\right)=\min \left\{\left\|\gamma_{1}\right\|,\left\|\gamma_{2}\right\|\right\}$.

(4) Suppose $\left\|\alpha_{1}\right\| \leq\left\|\alpha_{2}\right\|<\omega$ and $\alpha_{2} \stackrel{u}{\longrightarrow} \gamma$ is a norm-reducing path where $|u|=\left\|\alpha_{1}\right\|$ (and thus $\left.\|\gamma\|=\left\|\alpha_{2}\right\|-\left\|\alpha_{1}\right\|\right)$. Then for any $\delta_{1}, \delta_{2}$ we have

$\operatorname{EQLV}\left(\delta_{1}, \gamma \delta_{2}\right) \geq \operatorname{EQLV}\left(\alpha_{1} \delta_{1}, \alpha_{2} \delta_{2}\right)-\left\|\alpha_{1}\right\|$.

(5) $\operatorname{EQLv}\left(\sigma_{1} \mu, \sigma_{2} \mu\right)=\operatorname{EQLv}\left(\sigma_{1}, \sigma_{2}\right)+\|\mu\|$.

Proof. Points 1,2,3 are easy to observe.

(4) If $\left\|\alpha_{1} \delta_{1}\right\| \neq\left\|\alpha_{2} \delta_{2}\right\|$ then $\left\|\delta_{1}\right\|=\left\|\alpha_{1} \delta_{1}\right\|-\left\|\alpha_{1}\right\| \neq\left\|\alpha_{2} \delta_{2}\right\|-\left\|\alpha_{1}\right\|=\left\|\gamma \delta_{2}\right\|$, and (3) implies

$$
\begin{gathered}
\operatorname{EQLV}\left(\delta_{1}, \gamma \delta_{2}\right)=\min \left\{\left\|\delta_{1}\right\|,\left\|\gamma \delta_{2}\right\|\right\}=\min \left\{\left\|\alpha_{1} \delta_{1}\right\|,\left\|\alpha_{1} \gamma \delta_{2}\right\|\right\}-\left\|\alpha_{1}\right\|= \\
\min \left\{\left\|\alpha_{1} \delta_{1}\right\|,\left\|\alpha_{2} \delta_{2}\right\|\right\}-\left\|\alpha_{1}\right\|=\operatorname{EQLV}\left(\alpha_{1} \delta_{1}, \alpha_{2} \delta_{2}\right)-\left\|\alpha_{1}\right\| .
\end{gathered}
$$

We now assume $\left\|\alpha_{1} \delta_{1}\right\|=\left\|\alpha_{2} \delta_{2}\right\|$ (hence also $\left\|\delta_{1}\right\|=\left\|\gamma \delta_{2}\right\|$ ) and we contradict the assumption

$$
\operatorname{EQLv}\left(\delta_{1}, \gamma \delta_{2}\right)<\operatorname{EQLv}\left(\alpha_{1} \delta_{1}, \alpha_{2} \delta_{2}\right)-\left\|\alpha_{1}\right\|
$$

as follows. By Prop. 3.2(2), the norm-reducing path $\alpha_{2} \delta_{2} \stackrel{u}{\longrightarrow} \gamma \delta_{2}$ has a matching path $\alpha_{1} \delta_{1} \stackrel{u}{\longrightarrow} \sigma \delta_{1}$ where $\operatorname{EQLV}\left(\sigma \delta_{1}, \gamma \delta_{2}\right) \geq \operatorname{EQLv}\left(\alpha_{1} \delta_{1}, \alpha_{2} \delta_{2}\right)-\left\|\alpha_{1}\right\|$. Hence $\sigma \neq \varepsilon$ (i.e., $\alpha_{1} \stackrel{u}{\longrightarrow} \sigma$ is not norm-reducing), and thus $\left\|\sigma \delta_{1}\right\|>\left\|\gamma \delta_{2}\right\|$, which entails $\operatorname{EQLv}\left(\sigma \delta_{1}, \gamma \delta_{2}\right)=\left\|\gamma \delta_{2}\right\|$. Since $\operatorname{EQLv}\left(\delta_{1}, \gamma \delta_{2}\right) \geq\left\|\gamma \delta_{2}\right\|$ (by (2)), by (4.1) we would get a contradiction:

$$
\left\|\gamma \delta_{2}\right\| \leq \operatorname{EQLv}\left(\delta_{1}, \gamma \delta_{2}\right)<\operatorname{EQLv}\left(\alpha_{1} \delta_{1}, \alpha_{2} \delta_{2}\right)-\left\|\alpha_{1}\right\| \leq \operatorname{EQLv}\left(\sigma \delta_{1}, \gamma \delta_{2}\right)=\left\|\gamma \delta_{2}\right\| .
$$

(5) The equality surely holds if $\|\mu\|=\omega$ (in which case $\sigma_{1} \mu \sim U \sim \sigma_{2} \mu$ ) or if $\sigma_{1} \sim \sigma_{2}$; we thus further assume that $\mu$ is normed and $\operatorname{EQLv}\left(\sigma_{1}, \sigma_{2}\right)<\omega$.

- We show $\operatorname{EQLv}\left(\sigma_{1} \mu, \sigma_{2} \mu\right) \leq \operatorname{EQLv}\left(\sigma_{1}, \sigma_{2}\right)+\|\mu\|$ by induction on $\operatorname{EQLv}\left(\sigma_{1}, \sigma_{2}\right)$. If $\operatorname{EQLv}\left(\sigma_{1}, \sigma_{2}\right)=0$ then precisely one of $\sigma_{1}, \sigma_{2}$ is $\varepsilon$, and $\operatorname{EQLv}\left(\sigma_{1} \mu, \sigma_{2} \mu\right)=\|\mu\|$. If $\operatorname{EQLv}\left(\sigma_{1}, \sigma_{2}\right)=n+1$ (which entails $\sigma_{1} \neq \varepsilon, \sigma_{2} \neq \varepsilon$ ) then by Prop. 3.2(1,2) there are some transitions $\sigma_{1} \stackrel{a}{\longrightarrow} \tau_{1}$ and $\sigma_{2} \stackrel{a}{\longrightarrow} \tau_{2}$ such that 
(1) $\operatorname{EQLv}\left(\tau_{1}, \tau_{2}\right)<\operatorname{EQLv}\left(\sigma_{1}, \sigma_{2}\right)$, and

(2) $\operatorname{EQLv}\left(\tau_{1} \mu, \tau_{2} \mu\right) \geq \operatorname{EQLv}\left(\sigma_{1} \mu, \sigma_{2} \mu\right)-1$.

Since $\operatorname{EQLv}\left(\tau_{1} \mu, \tau_{2} \mu\right) \leq \operatorname{EQLv}\left(\tau_{1}, \tau_{2}\right)+\|\mu\|$ by the induction hypothesis, we have $\operatorname{EQLv}\left(\sigma_{1} \mu, \sigma_{2} \mu\right) \leq 1+\operatorname{EQLv}\left(\tau_{1}, \tau_{2}\right)+\|\mu\| \leq \operatorname{EQLv}\left(\sigma_{1}, \sigma_{2}\right)+\|\mu\|$.

- We show $\operatorname{EQLv}\left(\sigma_{1} \mu, \sigma_{2} \mu\right) \geq \operatorname{EQLv}\left(\sigma_{1}, \sigma_{2}\right)+\|\mu\|$ by induction on $\operatorname{EQLv}\left(\sigma_{1} \mu, \sigma_{2} \mu\right)$, excluding the trivial case $\operatorname{EQLv}\left(\sigma_{1} \mu, \sigma_{2} \mu\right)=\omega$.

The case $\operatorname{EQLv}\left(\sigma_{1} \mu, \sigma_{2} \mu\right)=0$ is trivial since it entails $\mu=\varepsilon$.

If $\operatorname{EQLv}\left(\sigma_{1} \mu, \sigma_{2} \mu\right)=n+1$ then at most one of $\sigma_{1}, \sigma_{2}$ can be empty. If we have $\sigma_{j}=\varepsilon(j \in\{1,2\})$ then $\operatorname{EQLv}\left(\sigma_{1}, \sigma_{2}\right)=0$ and $\operatorname{EQLv}\left(\sigma_{1} \mu, \sigma_{2} \mu\right)=\|\mu\|$ (by $\left.(3)\right)$; the claim thus holds. If both $\sigma_{1}, \sigma_{2}$ are nonempty then by Prop. 3.2 $(1,2)$ there are some transitions $\sigma_{1} \stackrel{a}{\longrightarrow} \tau_{1}$ and $\sigma_{2} \stackrel{a}{\longrightarrow} \tau_{2}$ such that

(1) $\operatorname{EQLv}\left(\tau_{1} \mu, \tau_{2} \mu\right)<\operatorname{EQLv}\left(\sigma_{1} \mu, \sigma_{2} \mu\right)$, and

(2) $\operatorname{EQLv}\left(\tau_{1}, \tau_{2}\right) \geq \operatorname{EQLv}\left(\sigma_{1}, \sigma_{2}\right)-1$.

Since $\operatorname{EQLv}\left(\tau_{1} \mu, \tau_{2} \mu\right) \geq \operatorname{EQLv}\left(\tau_{1}, \tau_{2}\right)+\|\mu\|$ by the induction hypothesis, we have $\operatorname{EQLv}\left(\sigma_{1} \mu, \sigma_{2} \mu\right) \geq 1+\operatorname{EQLv}\left(\tau_{1}, \tau_{2}\right)+\|\mu\| \geq \operatorname{EQLv}\left(\sigma_{1}, \sigma_{2}\right)+\|\mu\|$.

We now prove the announced theorem. Let us recall that the value $M_{r h s}$ (in Def. 3.5) is bounded by an exponential function of the size of $\mathcal{G}$ (by Prop. 3.6).

Theorem 4.5. Let $\mathcal{G}=(\mathcal{N}, \mathcal{A}, \mathcal{R})$ be a normed BPA system, and $M_{\text {rhs }}=\max \{\|\alpha\| ;$ there is a rule $A \stackrel{a}{\longrightarrow} \alpha$ in $\mathcal{R}\}$. If $\alpha_{1} \nsim \alpha_{2}$ then $\operatorname{EQLv}\left(\alpha_{1}, \alpha_{2}\right) \leq \min \left\{\left\|\alpha_{1}\right\|,\left\|\alpha_{2}\right\|\right\}+\operatorname{card}(\mathcal{N})^{2} \cdot M_{\text {rhs }}$.

Proof. If $\left\|\alpha_{1}\right\|<\left\|\alpha_{2}\right\|$ then $\operatorname{EQLv}\left(\alpha_{1}, \alpha_{2}\right) \leq\left\|\alpha_{1}\right\|$, as we noted in Prop. 3.4(1) for general BPA systems. We thus consider $\alpha_{1} \nsim \alpha_{2}$ where $\left\|\alpha_{1}\right\|=\left\|\alpha_{2}\right\|$, and we will work in the LTS $\mathcal{L}_{\mathcal{G}^{\prime}}$, where $\mathcal{G}^{\prime}$ is the completion of $\mathcal{G}$; the achieved upper bound will be also valid for $\mathcal{L}_{\mathcal{G}}$ by Prop. 4.3(1,3). We will construct a sequence

$$
\left(\rho_{1}, \rho_{1}^{\prime}, \mu_{1}\right),\left(\rho_{2}, \rho_{2}^{\prime}, \mu_{2}\right), \ldots,\left(\rho_{m}, \rho_{m}^{\prime}, \mu_{m}\right)
$$

where $\left(\rho_{1}, \rho_{1}^{\prime}, \mu_{1}\right)=\left(\alpha_{1}, \alpha_{2}, \varepsilon\right)$. We use a slightly modified process of constructing the sequence (3.2) in the proof of Lemma 3.11 in Subsection 3.4. Given $\left(\rho_{i}, \rho_{i}^{\prime}, \mu_{i}\right)$, where $\left\|\rho_{i}\right\|=\left\|\rho_{i}^{\prime}\right\|<\omega$ and $\operatorname{EQLv}\left(\rho_{i} \mu_{i}, \rho_{i}^{\prime} \mu_{i}\right)=\operatorname{EQLv}\left(\rho_{i}, \rho_{i}^{\prime}\right)+\|\mu\|<\omega$, we now construct $\left(\rho_{i+1}, \rho_{i+1}^{\prime}, \mu_{i+1}\right)$. As in the proof of Lemma 3.11, we write

$$
\rho_{i}=A_{1} \delta_{1}, \rho_{i}^{\prime}=A_{2} \delta_{2}
$$

where $\left\|A_{1}\right\| \leq\left\|A_{2}\right\|$ and we assume that the pair $\left(A_{1}, A_{2}\right)$ has a fixed norm-reducing path $A_{2} \stackrel{u}{\longrightarrow} \gamma$ such that $\left\|A_{1} \gamma\right\|=\left\|A_{2}\right\|$; we thus also have $\left\|\delta_{1}\right\|=\left\|\gamma \delta_{2}\right\|$.

(1) $\left(\rho_{i}, \rho_{i}^{\prime}\right)=\left(A_{1} \gamma, A_{2}\right)$, i.e. $\delta_{1}=\gamma$ and $\delta_{2}=\varepsilon$ in (4.3):

By Prop. $3.2(1,2)$ there are rules $A_{1} \stackrel{a}{\longrightarrow} \sigma_{1}, A_{2} \stackrel{a}{\longrightarrow} \sigma_{2}$ such that $\operatorname{EQLv}\left(\sigma_{1} \gamma, \sigma_{2}\right)=$ $\operatorname{EQLv}\left(A_{1} \gamma, A_{2}\right)-1$ (and where we thus do not have $\sigma_{1}=\sigma_{2}=U$ ). We put

$$
\left(\rho_{i+1}, \rho_{i+1}^{\prime}, \mu_{i+1}\right)=\left(\sigma_{1} \gamma, \sigma_{2}, \mu_{i}\right)
$$

and we note (by recalling that $\left.\operatorname{EQLv}\left(\rho \mu, \rho^{\prime} \mu\right)=\operatorname{EQLv}\left(\rho, \rho^{\prime}\right)+\|\mu\|\right)$ :

- $\operatorname{EQLv}\left(\rho_{i+1}, \rho_{i+1}^{\prime}\right)=\operatorname{EQLv}\left(\rho_{i}, \rho_{i}^{\prime}\right)-1$,

- $\operatorname{EQLv}\left(\rho_{i+1} \mu_{i+1}, \rho_{i+1}^{\prime} \mu_{i+1}\right)=\operatorname{EQLv}\left(\rho_{i} \mu_{i}, \rho_{i}^{\prime} \mu_{i}\right)-1$,

- $\min \left\{\left\|\rho_{i+1} \mu_{i+1}\right\|,\left\|\rho_{i+1}^{\prime} \mu_{i+1}\right\|\right\} \leq\left\|\rho_{i} \mu_{i}\right\|+M_{r h s}-1$.

We have the following two possibilities.

(a) If $\left\|\sigma_{1} \gamma\right\| \neq\left\|\sigma_{2}\right\|$ then $\left\|\rho_{i+1}\right\| \neq\left\|\rho_{i+1}^{\prime}\right\|$ and the sequence (4.2) is completed, i.e. $i+1=m$. In this case 
$\operatorname{EQLv}\left(\rho_{m} \mu_{m}, \rho_{m}^{\prime} \mu_{m}\right)=\min \left\{\left\|\rho_{m} \mu_{m}\right\|,\left\|\rho_{m}^{\prime} \mu_{m}\right\|\right\}$.

(b) If $\left\|\sigma_{1} \gamma\right\|=\left\|\sigma_{2}\right\|$ then $\left\|\rho_{i+1} \mu_{i+1}\right\|=\left\|\rho_{i+1}^{\prime} \mu_{i+1}\right\|<\omega$.

(2) $\left(\rho_{i}, \rho_{i}^{\prime}\right)=\left(A_{1} \delta_{1}, A_{2} \delta_{2}\right) \neq\left(A_{1} \gamma, A_{2}\right)$ and $\operatorname{EQLv}\left(A_{1} \gamma \delta_{2}, A_{2} \delta_{2}\right)=\operatorname{EQLv}\left(A_{1} \delta_{1}, A_{2} \delta_{2}\right)$ :

We put

and note:

$$
\left(\rho_{i+1}, \rho_{i+1}^{\prime}, \mu_{i+1}\right)=\left(A_{1} \gamma, A_{2}, \delta_{2} \mu_{i}\right)
$$

- $\operatorname{EQLv}\left(\rho_{i+1}, \rho_{i+1}^{\prime}\right)=\operatorname{EQLv}\left(\rho_{i}, \rho_{i}^{\prime}\right)-\left\|\delta_{2}\right\| \leq \operatorname{EQLv}\left(\rho_{i}, \rho_{i}^{\prime}\right)$,

- $\operatorname{EQLv}\left(\rho_{i+1} \mu_{i+1}, \rho_{i+1}^{\prime} \mu_{i+1}\right)=\operatorname{EQLv}\left(\rho_{i} \mu_{i}, \rho_{i}^{\prime} \mu_{i}\right)$,

- $\left\|\rho_{i+1} \mu_{i+1}\right\|=\left\|\rho_{i+1}^{\prime} \mu_{i+1}\right\|=\left\|\rho_{i} \mu_{i}\right\|=\left\|\rho_{i}^{\prime} \mu_{i}\right\|$.

Moreover, for $i+1$ the above case (1) will apply.

(3) $\operatorname{EQLv}\left(A_{1} \gamma \delta_{2}, A_{2} \delta_{2}\right) \neq \operatorname{EQLv}\left(A_{1} \delta_{1}, A_{2} \delta_{2}\right)$ (which entails $\left.\left(\rho_{i}, \rho_{i}^{\prime}\right) \neq\left(A_{1} \gamma, A_{2}\right)\right)$ :

We thus have $\operatorname{EQLv}\left(A_{1} \delta_{1}, A_{1} \gamma \delta_{2}\right) \leq \operatorname{EQLv}\left(A_{1} \delta_{1}, A_{2} \delta_{2}\right)$, by Prop. $3.2(3)$.

Since $\operatorname{EQLv}\left(A_{1} \delta_{1}, A_{1} \gamma \delta_{2}\right) \geq\left\|A_{1}\right\|+\operatorname{EQLv}\left(\delta_{1}, \gamma \delta_{2}\right)$ (by Prop. 3.4 (3)), we get

$$
\operatorname{EQLv}\left(\delta_{1}, \gamma \delta_{2}\right) \leq \operatorname{EQLv}\left(A_{1} \delta_{1}, A_{1} \gamma \delta_{2}\right)-\left\|A_{1}\right\| \leq \operatorname{EQLv}\left(A_{1} \delta_{1}, A_{2} \delta_{2}\right)-\left\|A_{1}\right\| .
$$

On the other hand, Prop. 4.4(4) implies

$$
\operatorname{EQLv}\left(\delta_{1}, \gamma \delta_{2}\right) \geq \operatorname{EQLv}\left(A_{1} \delta_{1}, A_{2} \delta_{2}\right)-\left\|A_{1}\right\|
$$

Hence $\operatorname{EQLv}\left(\delta_{1}, \gamma \delta_{2}\right)=\operatorname{EQLv}\left(A_{1} \delta_{1}, A_{2} \delta_{2}\right)-\left\|A_{1}\right\|$. We put

$$
\left(\rho_{i+1}, \rho_{i+1}^{\prime}, \mu_{i+1}\right)=\left(\delta_{1}, \gamma \delta_{2}, \mu_{i}\right)
$$

and note:

- $\operatorname{EQLv}\left(\rho_{i+1}, \rho_{i+1}^{\prime}\right)=\operatorname{EQLv}\left(\rho_{i}, \rho_{i}^{\prime}\right)-\left\|A_{1}\right\|$

- $\operatorname{EQLv}\left(\rho_{i+1} \mu_{i+1}, \rho_{i+1}^{\prime} \mu_{i+1}\right)=\operatorname{EQLv}\left(\rho_{i} \mu_{i}, \rho_{i}^{\prime} \mu_{i}\right)-\left\|A_{1}\right\|$,

- $\left\|\rho_{i+1} \mu_{i+1}\right\|=\left\|\rho_{i+1}^{\prime} \mu_{i+1}\right\|=\left\|\rho_{i} \mu_{i}\right\|-\left\|A_{1}\right\|$.

As in Subsection 3.4, due to eq-level decreasing the case (1) cannot apply more than $\operatorname{card}(\mathcal{N})^{2}$ times, and the construction must end eventually, with $\left\|\rho_{m} \mu_{m}\right\| \neq\left\|\rho_{m}^{\prime} \mu_{m}\right\|$ arising in (1a). Let us now put

$$
e_{i}=\operatorname{EQLv}\left(\rho_{i} \mu_{i}, \rho_{i}^{\prime} \mu_{i}\right) \text {, and } d_{i}=e_{i}-\min \left\{\left\|\rho_{i} \mu_{i}\right\|,\left\|\rho_{i}^{\prime} \mu_{i}\right\|\right\} .
$$

In fact, in (1a) we noted that $d_{m}=0$. If (2) or (3) applies to $i$ then we obviously have $d_{i}=d_{i+1}$. We can also easily check that if (1) applies to $i$ then

$$
e_{i+1}-\min \left\{\left\|\rho_{i+1} \mu_{i+1}\right\|,\left\|\rho_{i+1}^{\prime} \mu_{i+1}\right\|\right\} \geq\left(e_{i}-1\right)-\left(\min \left\{\left\|\rho_{i} \mu_{i}\right\|,\left\|\rho_{i}^{\prime} \mu_{i}\right\|\right\}+M_{r h s}-1\right) .
$$

This yields $d_{i+1} \geq d_{i}-M_{r h s}$, hence $d_{i} \leq d_{i+1}+M_{r h s}$. We thus deduce $d_{1} \leq \operatorname{card}(\mathcal{N})^{2} \cdot M_{r h s}$, i.e., $e_{1} \leq \min \left\{\left\|\rho_{1} \mu_{1}\right\|,\left\|\rho_{1}^{\prime} \mu_{1}\right\|\right\}+\operatorname{card}(\mathcal{N})^{2} \cdot M_{r h s}$. Since $\left(\rho_{1}, \rho_{1}^{\prime}, \mu_{1}\right)=\left(\alpha_{1}, \alpha_{2}, \varepsilon\right)$, we get

$$
\operatorname{EQLv}\left(\alpha_{1}, \alpha_{2}\right) \leq \min \left\{\left\|\alpha_{1}\right\|,\left\|\alpha_{2}\right\|\right\}+\operatorname{card}(\mathcal{N})^{2} \cdot M_{r h s} .
$$




\section{AdDitional REMARKS}

Lemma 3.14 shows that the pairs $(\alpha, \beta)$ where $\alpha \sim \beta, \alpha, \beta$ have $\mathcal{E}$-bounded cycles, and $\operatorname{SIZE}\left(\alpha_{p}, \beta_{p}\right) \leq 2 M+\mathcal{E}$ create a basis for $\mathcal{G}$, similar to the bisimulation base of [6] but with explicit regular strings. We could construct the basis by a standard coinductive approach (building a sequence of decreasing overapproximations). Each of the pairs in the basis fits into exponential space, and their number is thus at most double exponential.

Among the related topics for future research, the obvious one is the question how to close the gap between ExPTIME and 2-ExPTIME for bisimilarity on BPA. Other examples of research topics follow from the fact that BPA processes can be viewed as being generated by pushdown automata with a single control state and no $\varepsilon$-transitions. Sénizergues [18] showed the decidability of bisimilarity for general pushdown processes where $\varepsilon$-transitions are deterministic and popping; it seems interesting to explore the decomposition approach here as well, using regular terms (as in [12]). One indication that this more general problem is also more complicated is a recent announcement [2] that its computational complexity is nonelementary. We can also mention that bisimilarity of pushdown processes with nondeterministic popping $\varepsilon$-transitions is undecidable [13]; this was shown by using so called "Defender's Forcing", which was recently also used to show undecidability for $2^{\text {nd }}$-order pushdown processes with no $\varepsilon$-transitions [4]. The decidability question for BPA with $\varepsilon$ transitions (i.e., the weak bisimilarity problem for BPA) is still open.

Acknowledgement. The author cordially thanks to anonymous reviewers for helpful comments and suggestions.

\section{REFERENCES}

[1] J. Baeten, J. Bergstra, and J. Klop. Decidability of bisimulation equivalence for processes generating context-free languages. J.ACM, 40(3):653-682, 1993.

[2] M. Benedikt, S. Göller, S. Kiefer, and A. S. Murawski. Bisimilarity of pushdown systems is nonelementary. CoRR, abs/1210.7686, 2012.

[3] S. Böhm, S. Göller, and P. Jančar. Bisimilarity of one-counter processes is PSPACE-complete. In CONCUR 2010 - Concurrency Theory, volume 6269 of LNCS, pages 177-191. Springer-Verlag, 2010.

[4] C. H. Broadbent and S. Göller. On bisimilarity of higher-order pushdown automata: Undecidability at order two. In FSTTCS 2012, volume 18 of LIPIcs, pages 160-172. Schloss Dagstuhl - Leibniz-Zentrum fuer Informatik, 2012.

[5] O. Burkart, D. Caucal, F. Moller, and B. Steffen. Verification on infinite structures. In J. Bergstra, A. Ponse, and S. Smolka, editors, Handbook of Process Algebra, pages 545-623. North-Holland, 2001.

[6] O. Burkart, D. Caucal, and B. Steffen. An elementary bisimulation decision procedure for arbitrary context-free processes. In Proc. of MFCS'95, volume 969 of LNCS, pages 423-433. Springer, 1995.

[7] A. K. Chandra, D. C. Kozen, and L. J. Stockmeyer. Alternation. J. ACM, 28(1):114-133, 1981.

[8] S. Christensen, H. Hüttel, and C. Stirling. Bisimulation equivalence is decidable for all context-free processes. Inf. Comput., 121(2):143-148, 1995.

[9] W. Czerwiński and S. Lasota. Fast equivalence-checking for normed context-free processes. In Proc. FSTTCS'10, volume 8 of LIPIcs. Schloss Dagstuhl - Leibniz-Zentrum fuer Informatik, 2010.

[10] Y. Hirshfeld, M. Jerrum, and F. Moller. A polynomial algorithm for deciding bisimilarity of normed context-free processes. Theor. Comput. Sci., 158:143-159, 1996.

[11] P. Jančar. Strong bisimilarity on basic parallel processes is PSPACE-complete. In Proc. LICS 2003, pages 218-227. IEEE Computer Society, 2003.

[12] P. Jančar. Decidability of DPDA language equivalence via first-order grammars. In Proc. LICS 2012. IEEE Computer Society, 2012.

[13] P. Jančar and J. Srba. Undecidability of bisimilarity by Defender's forcing. J. ACM, 55(1), 2008. 
[14] M. Jurdzinski, J. Sproston, and F. Laroussinie. Model checking probabilistic timed automata with one or two clocks. Logical Methods in Computer Science, 4(3), 2008.

[15] S. Kiefer. BPA bisimilarity is EXPTIME-hard. Inf. Proc. Letters, 113(4):101-106, 2013.

[16] A. Kučera and R. Mayr. On the complexity of checking semantic equivalences between pushdown processes and finite-state processes. Inf. Comput., 208(7):772-796, 2010.

[17] R. Mayr. Weak bisimilarity and regularity of context-free processes is exptime-hard. Theor. Comput. Sci., 330(3):553-575, 2005.

[18] G. Sénizergues. The bisimulation problem for equational graphs of finite out-degree. SIAM J.Comput., 34(5):1025-1106, 2005.

[19] J. Srba. Strong bisimilarity of simple process algebras: complexity lower bounds. Acta Inf., 39(6-7):469499, 2003.

[20] J. Srba. Beyond language equivalence on visibly pushdown automata. Logical Methods in Computer Science, 5(1), 2009. 